\title{
CONTENTS OF VOLUME 84, 2020
}

\section{Message From the Editor-in-Chief}

1. Big Changes for the Circulation Journal

Toyoaki Murohara

2. Editorial Statistics and Best Reviewers Award for 2019

Toyoaki Murohara

3. Circulation Journal Awards for the Year 2019

Toyoaki Murohara

4. Message From the Editor-in-Chief

Toyoaki Murohara

5. Message From the Editor-in-Chief Toyoaki Murohara

6. Greetings From the New Editor-in-Chief Toshihisa Anzai

7. Message From the Editor-in-Chief Toshihisa Anzai

8. Message From the Editor-in-Chief Toshihisa Anzai

\section{In Memoriam}

1. Tribute to Dr. Tomisaku Kawasaki - Discoverer of Kawasaki Disease and a Great Pediatrician Hirohisa Kato

\section{JCS Statement}

1. A Statement on the Appropriate Administration of Tafamidis in Patients With Transthyretin Cardiac Amyloidosis Jin Endo, Motoaki Sano, Yasuhiro Izumiya, Kenichi Tsujita, Kazufumi Nakamura, Nobuhiro Tahara, Koichiro Kuwahara, Takayuki Inomata, Mitsuharu Ueda, Yoshiki Sekijima, Yukio Ando, Hiroyuki Tsutsui, Mitsuaki Isobe, Keiichi Fukuda

2. Japanese Heart Failure Society 2018 Scientific Statement on Nutritional Assessment and Management in Heart Failure Patients

Kazuhiro Yamamoto, Miyuki Tsuchihashi-Makaya, Yoshiharu Kinugasa, Yuki Iida, Kentaro Kamiya, Yasuki Kihara, Yuji Kono, Yukihito Sato, Norio Suzuki, Harumi Takeuchi, Taiki Higo, Yasushi Miyazawa, Isao Miyajima, Akira Yamashina, Katsushi Yoshita, Koichi Washida, Masafumi Kuzuya, Tetsuya Takahashi, Yutaka Nakaya, Naoyuki Hasebe, Hiroyuki Tsutsui on behalf of The Japanese Heart Failure Society, Expert Consensus Writing Committee

\section{Reviews}

1. Uremic Toxin-Targeting as a Therapeutic Strategy for Preventing Cardiorenal Syndrome Kensei Taguchi, Bertha C. Elias, Craig R. Brooks, Seiji Ueda, Kei Fukami

2. The Asia-Pacific Society of Cardiology (APSC) Expert Committee Consensus Recommendations for Assessment of Suspected Acute Coronary Syndrome Using High-Sensitivity Cardiac Troponin T in the Emergency Department

Wei Chieh Jack Tan, Kenji Inoue, Laila AbdelWareth, Evangelos Giannitsis, Sazzli Kasim, Masayuki Shiozaki, Tar Choon Aw, Federick Cheng, Ho Thuong Dung, Yi-Heng Li, Swee Han Lim, Antonia Anna Lukito, Martin Than, Fang-Yeh Chu, Tom Devasia, Chien-Chang Lee, Arintaya Phrommintikul, Jong-Chan Youn, Derek P. Chew

3. Strengths and Opportunities of Network Medicine in Cardiovascular Diseases

Giuditta Benincasa, Raffaele Marfella, Nunzia Della Mura, Concetta Schiano, Claudio Napoli

4. Beautiful Harmony of the Japanese Precious Healthcare Legacies for the New Imperial Era

Hidehiro Kaneko, Hiroyuki Morita, Issei Komuro

5. Mechanical Circulatory Support for Patients With Adult Congenital Heart Disease Maruti Haranal, Shuhua Luo, Osami Honjo

6. Cardiac Arrhythmias in Autoimmune Diseases Monika Gawałko, Paweł Balsam, Piotr Lodziński, Marcin Grabowski, Bartosz Krzowski, Grzegorz Opolski, Jędrzej Kosiuk

7. Lipoprotein(a) and Cardiovascular Diseases - Revisited Albert Youngwoo Jang, Seung Hwan Han, Il Suk Sohn, Pyung Chun Oh, Kwang Kon Koh 
8. Electrophysiological Procedures in Patients With Coagulation Disorders - A Systemic Review Bartosz Krzowski, Paweł Balsam, Michał Peller, Piotr Lodziński, Marcin Grabowski, Joanna Drozd-Sokołowska, Grzegorz Basak, Monika Gawałko, Grzegorz Opolski, Jedrzej Kosiuk

9. Three-Dimensional Echocardiography - Role in Clinical Practice and Future Directions Kazuaki Tanabe

10. Heart Failure in Atrial Fibrillation - An Update on Clinical and Echocardiographic Implications Naoki Taniguchi, Yoko Miyasaka, Yoshinobu Suwa, Shoko Harada, Eri Nakai, Ichiro Shiojima

11. Hypertrophic Cardiomyopathy - A Heterogeneous and Lifelong Disease in the Real World Hiroaki Kitaoka, Toru Kubo, Yoshinori L. Doi

12. Cancer Therapeutics-Related Cardiac Dysfunction - Insights From Bench and Bedside of Onco-Cardiology Hiroshi Kadowaki, Hiroshi Akazawa, Junichi Ishida, Issei Komuro

13. Management of Atrial Fibrillation in COVID-19 Pandemic Yu-Feng Hu, Wen-Han Cheng, Yuan Hung, Wen-Yu Lin, Tze-Fan Chao, Jo-Nan Liao, Yenn-Jiang Lin, Wei-Shiang Lin, Yi-Jen Chen, Shih-Ann Chen

14. Future Directions of Therapeutic Vaccines for Chronic Diseases Ryo Nakamaru, Hironori Nakagami, Hiromi Rakugi, Ryuichi Morishita

15. Current Understanding of the Role of Frailty in Cardiovascular Disease Yoshihiro Uchikado, Yoshiyuki Ikeda, Mitsuru Ohishi

16. Pluripotent Stem Cells for Cardiac Regeneration - Current Status, Challenges, and Future Perspectives Yuji Shiba

\section{Editorials}

1. Advantages and Emerging Problems of Novel Treatments for Transthyretin Cardiac Amyloidosis Takahiro Okumura, Toyoaki Murohara

2. Can a New Algorithm of Cardiac Resynchronization Therapy (Adaptive CRT) Expand Its Utility? Takashi Kurita

3. Optical Technology Stepping Forward - Utility of Intracoronary Optical Coherence Tomography Myong Hwa Yamamoto, Toshiro Shinke

4. Individualized Duration of Dual Antiplatelet Therapy Guided by Risk Scores - Ready for Prime Time? Sergio Buccheri, Stefan James

5. Local Epicardial Adipose Tissue Deposits and Left Ventricular Diastolic Function in Patients With Preserved Left Ventricular Ejection Fraction

Toshiaki Nakajima, Shigeru Toyoda, Teruo Inoue

6. Should Patients Choose Lung Transplantation or Optimal Drug Therapy? - The Clinical Situation in the Treatment of Pulmonary Arterial Hypertension in Japan -

Hisataka Maki

7. Importance of Atrial Fibrillation in Heart Failure Patients With Preserved Ejection Fraction Without Coronary Artery Disease Hisanori Kanazawa, Kenichi Tsujita

8. High-Sensitivity C-Reactive Protein and Bleeding Events in Atrial Fibrillation Patients Treated With Direct Oral Anticoagulants

Tetsuji Shinohara, Naohiko Takahashi

9. Cardiac Rehabilitation as Therapeutic Strategy After Coronary Artery Bypass Grafting Hideo Izawa

10. Early Lifestyle Modification Is an Essential Step in Telemedicine for Heart Failure Norimichi Koitabashi, Masaru Obokata, Masahiko Kurabayashi

11. Major Influence of Diabetes on Hospitalization for Heart Failure in Patients With Ischemic Heart Diseases Tomohisa Nagoshi

12. Nitroglycerin Tolerance in Patients With ALDH2 Variant Kyung-Duk Min, Masafumi Kitakaze

13. Non-Fasting Triglyceride Levels as a Superior Predictor of Cardiovascular Disease Hyemoon Chung, Weon Kim

14. Clinical Significance of Heart Rate in Acute Cardiovascular Diseases - Simple But Meaningful Naoki Sato

15. A Diversified Approach for the Prevention of Pediatric Sudden Cardiovascular Death in Japan Taichi Kato

16. Are You Ready for Kaizen in Your Clinical Practice?

Sumio Yamada

17. Therapeutic Angiogenesis Using Bone Marrow Mononuclear Cell Transplantation - A New Standard Treatment for Thromboangiitis Obliterans? -

Rei Shibata, Shukuro Yamaguchi, Toyoaki Murohara 
18. National Health Insurance Database and Atrial Fibrillation Research

Eiichi Watanabe

19. Atrial Fibrillation With Valvular Heart Disease - New Insight Into Clinical Outcomes Sayaka Kurokawa, Yasuo Okumura

20. Prediction of Thromboembolism in Patients With Hypertrophic Cardiomyopathy Takahiro Okumura, Yuki Kimura, Toyoaki Murohara

21. No Epicardial Fat, No Plaque Rupture Sho Torii, Gaku Nakazawa

22. Should Transcatheter Aortic Valve Implantation Be the First-Choice Treatment? An Important Step Forward Kentaro Hayashida

23. Prediction and Detection of Atrial Fibrillation After Catheter Ablation Yu-ki Iwasaki, Wataru Shimizu

24. Measuring Thrombogenicity in ST-Elevation Myocardial Infarction - Mechanistic Insights and Future Directions Sergio Buccheri

25. New-Onset Left Bundle Branch Block After Transcatheter Aortic Valve Implantation - Not a Harmless Bystander Shunji Hayashidani, Akira Shiose, Hiroyuki Tsutsui

26. Can Pharmacological Ischemic Preconditioning Affect Myocardial Ischemic Injury? Koji Ohashi, Noriyuki Ouchi, Toyoaki Murohara

27. How Should Rehabilitation Be Performed After Transcatheter Aortic Valve Replacement? Akihiro Tokushige, Mitsuru Ohishi

28. Rural Health Disparities in Japan - Urgent Need for Big Data Utilization and Health Service Research Toshiki Maeda, Hisatomi Arima

29. Diastolic Pressure Ratio and Resting Full Cycle Ratio Are Similar, But Not Exactly the Same Yasutsugu Shiono, Takashi Kubo, Takashi Akasaka

30. Prediction of Coronary Artery Disease by Measurement of Circulating MicroRNA-423-3p Levels Koh Ono

31. Management of Patients With Aortic Stenosis Requiring Non-Cardiac Surgery Yoshiyuki Tokuda, Akihiko Usui

32. 2-Year Outcomes of Left Atrial Appendage Occlusion With WATCHMAN in Japanese Atrial Fibrillation Patients Masahide Harada

33. Novel Players and a Target of Stem Cell Therapy for the Heart Oto Inoue, Kosei Yamaguchi, Masayuki Takamura

34. Direct Oral Anticoagulants for the Treatment of Cancer-Associated Venous Thromboembolism in Real-World Clinical Practice - Caution Regarding Substantial Frequency of Bleeding Complications Yutaka Hosoi

35. Risk of Pulmonary Arterial Hypertension During Qing-Dai Use for the Treatment of Ulcerative Colitis Shiro Adachi, Yoshihisa Nakano, Takahisa Kondo

36. Significance of Atrial Fibrillation Management Based on the Sequence of Atrial Fibrillation and Heart Failure Onset Yoshihiro Seo, Nobuyuki Ohte

37. Gastrointestinal Bleeding in Japanese Patients With Atrial Fibrillation - Non-Critical Site Bleeding, But With Poor Prognosis -

Hiro Yamasaki
38. What Is the Optimal Dual Antiplatelet Therapy Strategy After Percutaneous Coronary Intervention in the Era of New-Generation Drug-Eluting Stent? Hiroaki Yokoyama

9. Still a Long Way to Go in Treating Cardiogenic Shock in Acute Myocardial Infarction

Jeehoon Kang, Hyojeong Ahn, Hak Seung Lee, Hyun-Jai Cho
40. Identification of Patient-Related Factors in Stent Thrombosis Shun Kohsaka

41. What Is the Most Preferable Treatment Strategy for Patients With Non-ST-Elevation Acute Coronary Syndrome With Multivessel Disease? - A Long-Term Perspective Yohei Numasawa

42. Heart Failure and Cancer - A Comorbid Risk That Is No Longer Underestimated Shingo Kazama, Yasuko K. Bando, Toyoaki Murohara

43. Potential of Selexipag in Chronic Thromboembolic Pulmonary Hypertension Medical Therapy Takahisa Kondo, Yoshihisa Nakano, Shiro Adachi

44. Molecular Mechanisms of Binge Drinking-Induced Atrial Fibrillation Yukiomi Tsuji

45. Importance of Validating Guideline Recommendations Arthur A.M. Wilde 
1. A Multicenter Prospective Observational Cohort Study to Investigate the Effectiveness and Safety of Rivaroxaban in Japanese Venous Thromboembolism Patients (The J'xactly Study)

Yasuo Okumura, Ikuo Fukuda, Mashio Nakamura, Norikazu Yamada, Morimasa Takayama, Hideaki Maeda, Takeshi Yamashita, Takanori Ikeda, Makoto Mo, Takao Kobayashi, Akihiro Niwa, Hiroshi Matsuo, Hiroyoshi Yokoi, Masatoshi Koga, Tsutomu Yamazaki, Atsushi Hirayama on behalf of the J'xactly Investigators

\section{Original Articles}

Arrhythmia/Electrophysiology (30)

1. Efficacy of a Device-Based Continuous Optimization Algorithm for Patients With Cardiac Resynchronization Therapy

Nobuhiko Ueda, Takashi Noda, Kohei Ishibashi, Kenzaburo Nakajima, Naoya Kataoka, Tsukasa Kamakura, Mitsuru Wada, Kenichiro Yamagata, Yuko Inoue, Koji Miyamoto, Satoshi Nagase, Takeshi Aiba, Hideaki Kanzaki, Chisato Izumi, Teruo Noguchi, Satoshi Yasuda, Kengo Kusano

2. Left Ventricular Inflow Velocity Pattern in Patients With Symptomatic Premature Ventricular Contraction Satoshi Takahashi, Takanao Mine, Kenki Ashida, Hideyuki Kishima, Tohru Masuyama, Masaharu Ishihara

3. Open-Label Two-Dose Pilot Study of Landiolol for the Treatment of Atrial Fibrillation/Atrial Flutter in Caucasian Patients

Guenter Stix, Michael Wolzt, Hans Domanovits, Pavla Kadlecová, Bernhard Husch, Michaela Trebs, Juri Hodisch, Martin Unger, Guenther Krumpl

4. Age-Related Differences in the Clinical Characteristics and Treatment of Elderly Patients With Atrial Fibrillation in Japan - Insight From the ANAFIE (All Nippon AF In Elderly) Registry -

Ken-ichi Hiasa, Hidetaka Kaku, Hiroshi Inoue, Takeshi Yamashita, Masaharu Akao, Hirotsugu Atarashi, Yukihiro Koretsune, Ken Okumura, Wataru Shimizu, Takanori Ikeda, Kazunori Toyoda, Atsushi Hirayama, Masahiro Yasaka, Takenori Yamaguchi, Satoshi Teramukai, Tetsuya Kimura, Jumpei Kaburagi, Atsushi Takita, Hiroyuki Tsutsui

5. Differential Prognostic Impact of Atrial Fibrillation in Hospitalized Heart Failure Patients With Preserved Ejection Fraction According to Coronary Artery Disease Status - Report From the Japanese Nationwide Multicenter Registry -

Taro Temma, Toshiyuki Nagai, Masaya Watanabe, Rui Kamada, Yumi Takahashi, Hikaru Hagiwara, Taro Koya, Motoki Nakao, Kazunori Omote, Kiwamu Kamiya, Hiroyuki Iwano, Kazuhiro Yamamoto, Tsutomu Yoshikawa, Yoshihiko Saito, Toshihisa Anzai

6. High Predictive Value of Adenosine Provocation in Predicting Atrial Fibrillation Recurrence After Pulmonary Vein Isolation With Visually Guided Laser Balloon Compared With Radiofrequency Ablation

Ekrem Üçer, Sabine Fredersdorf, Joachim Seegers, Florian Poschenrieder, Christian Hauck, Lars Maier, Carsten Jungbauer

7. Persistent Systemic Inflammation Is Associated With Bleeding Risk in Atrial Fibrillation Patients Yuma Hamanaka, Yohei Sotomi, Akio Hirata, Tomoaki Kobayashi, Yasuhiro Ichibori, Nobuhiko Makino, Takaharu Hayashi, Yasushi Sakata, Atsushi Hirayama, Yoshiharu Higuchi

8. Impact of Box Isolation on Rotors and Multiple Wavelets in Persistent Atrial Fibrillation Koichiro Kumagai, Hideko Toyama, Takashi Ashihara

9. Transient Elevation of Glucose Increases Arrhythmia Susceptibility in Non-Diabetic Rat Trabeculae With NonUniform Contraction

Masahito Miura, Tetsuya Handoh, Yuhto Taguchi, Taiki Hasegawa, Yui Takahashi, Natsuki Morita, Ayana Matsumoto, Chiyohiko Shindoh, Haruka Sato

10. High Prevalence of Late-Appearing T-Wave in Patients With Long QT Syndrome Type 8 Megumi Fukuyama, Seiko Ohno, Junichi Ozawa, Koichi Kato, Takeru Makiyama, Yoshihisa Nakagawa, Minoru Horie

11. Trends in Prevalence of Non-Valvular Atrial Fibrillation and Anticoagulation Therapy in a Japanese Region - Analysis Using the National Health Insurance Database -

Noritomo Narita, Ken Okumura, Takahiko Kinjo, Joko Mikami, Katsuko Tsushima, Reiko Takahashi, Makiko Noro, Ayaka Hashimoto, Teiko Sasaki, Misaki Takaki, Emiko Ishidoya, Yurika Narita, Hideno Imai, Emiko Ono, Hirofumi Tomita

12. Impact of Valvular Heart Disease on Mortality, Thromboembolic and Cardiac Events in Japanese Patients With Atrial Fibrillation - The Fushimi AF Registry -

Kosuke Doi, Hisashi Ogawa, Kenjiro Ishigami, Syuhei Ikeda, Yuya Aono, Yasuhiro Hamatani, Akiko Fujino, Yoshimori An, Mitsuru Ishii, Moritake Iguchi, Nobutoyo Masunaga, Masahiro Esato, Hikari Tsuji, Hiromichi Wada, Koji Hasegawa, Mitsuru Abe, Masaharu Akao on behalf of the Fushimi AF Registry Investigators

13. Burden and Long Firing of Premature Atrial Contraction Early After Catheter Ablation Predict Late Recurrence of Atrial Fibrillation

Hiroyuki Inoue, Nobuaki Tanaka, Koji Tanaka, Yuichi Ninomiya, Yuko Hirao, Takafumi Oka, Masato Okada, Ryo Kitagaki, Kohtaro Takayasu, Yasushi Koyama, Atsunori Okamura, Katsuomi Iwakura, Kenshi Fujii, Yasushi Sakata, Koichi Inoue 
14. Reconduction After Second-Generation Cryoballoon-Based Pulmonary Vein Isolation - Impact of Different Ablation Strategies -

Christian-Hendrik Heeger, Enida Rexha, Sabrina Maack, Laura Rottner, Thomas Fink, Shibu Mathew, Tilman Maurer, Christine Lemeš, Andreas Rillig, Peter Wohlmuth, Bruno Reissmann, Roland Richard Tilz, Feifan Ouyang, Karl-Heinz Kuck, Andreas Metzner

15. Clinical Implications of Ventricular Repolarization Parameters on Long-Term Risk of Atrial Fibrillation - Longitudinal Follow-up Data From a General Ambulatory Korean Population Min Soo Cho, Gi-Byoung Nam, Yu Na Kim, Jun Kim, Kee-Joon Choi, You-Ho Kim

16. Anti-Factor Xa Activity of Standard and Japan-Specific Doses of Rivaroxaban in Thai Patients With Non-Valvular Atrial Fibrillation

Wanwarang Wongcharoen, Phongsathon Pacharasupa, Lalita Norasetthada, Siriluck Gunaparn, Arintaya Phrommintikul

17. Efficacy and Safety of Left Atrial Appendage Closure With WATCHMAN in Japanese Nonvalvular Atrial Fibrillation Patients - Final 2-Year Follow-up Outcome Data From the SALUTE Trial -

Kazutaka Aonuma, Hiro Yamasaki, Masato Nakamura, Takashi Matsumoto, Morimasa Takayama, Kenji Ando, Kenzo Hirao, Masahiko Goya, Yoshihiro Morino, Kentaro Hayashida, Kengo Kusano, Yutaka Gomi, Michael L. Main, Takahiro Uchida, Shigeru Saito

18. Magnesium Deficiency Causes Transcriptional Downregulation of Kir2.1 and Kv4.2 Channels in Cardiomyocytes Resulting in QT Interval Prolongation

Toru Shimaoka, Yan Wang, Masaki Morishima, Shinji Miyamoto, Katsushige Ono

9. Impact of Renal Dysfunction on Left Atrial Structural Remodeling and Recurrence After Catheter Ablation for Atrial Fibrillation - A Propensity Score Matching Analysis -

Yuya Takahashi, Takanori Yamaguchi, Akira Fukui, Toyokazu Otsubo, Kei Hirota, Yuki Kawano, Kana Nakashima, Mai Tahara, Takayuki Kitai, Atsushi Kawaguchi, Naohiko Takahashi, Koichi Node

20. Impact of the Temporal Relationship Between Atrial Fibrillation and Heart Failure on Prognosis After Ablation Aki Tsuji, Masaharu Masuda, Mitsutoshi Asai, Osamu Iida, Shin Okamoto, Takayuki Ishihara, Kiyonori Nanto, Takashi Kanda, Takuya Tsujimura, Yasuhiro Matsuda, Shota Okuno, Yosuke Hata, Toshiaki Mano

21. Gastrointestinal Bleeding From Oral Anticoagulant Therapy Among Japanese Patients With Atrial Fibrillation Identified From the SAKURA Atrial Fibrillation Registry

Nobuhiro Murata, Yasuo Okumura, Koichi Nagashima, Daisuke Fukamachi, Katsuaki Yokoyama, Naoya Matsumoto, Eizo Tachibana, Keiichiro Kuronuma, Koji Oiwa, Michiaki Matsumoto, Toshiaki Kojima, Shoji Hanada, Kazumiki Nomoto, Ken Arima, Fumiyuki Takahashi, Tomobumi Kotani, Yukitoshi Ikeya, Seiji Fukushima, Satoru Itou, Kunio Kondo, Masaaki Chiku, Yasumi Ohno, Motoyuki Onikura, Atsushi Hirayama for the SAKURA AF Registry Investigators

22. Impact of Chronic Kidney Disease Classification on New-Onset Atrial Fibrillation in the General Population - The TAMA MED Project-AF and CKD -

Eitaro Kodani, Tomohiro Kaneko, Hitomi Fujii, Hiroyuki Nakamura, Hajime Sasabe, Yutaka Tamura, Wataru Shimizu

23. Seasonal Variations in the Incidence of Ischemic Stroke, Extracranial and Intracranial Hemorrhage in Atrial Fibrillation Patients

Mitsunori Oida, Shinya Suzuki, Takuto Arita, Naoharu Yagi, Takayuki Otsuka, Mikio Kishi, Hiroaki Semba, Hiroto Kano, Shunsuke Matsuno, Yuko Kato, Tokuhisa Uejima, Yuji Oikawa, Satoshi Hoshino, Minoru Matsuhama, Tatsuya Inoue, Junji Yajima, Takeshi Yamashita

24. Renin-Angiotensin System Inhibitors for the Prevention of Atrial Fibrillation Recurrence After Ablation - A MetaAnalysis -

Long Peng, Zexiong Li, Yanting Luo, Xixiang Tang, Xing Shui, Xujing Xie, Zhenda Zheng, Ruimin Dong, Jinlai Liu, Jieming Zhu, Suhua Li

25. Role of Remote Monitoring in Detection of Atrial Arrhythmia, Stroke Reduction, and Use of Anticoagulation Therapy - A Systematic Review and Meta-Analysis Jia-Pei Jang, Hui-Ting Lin, Yu-Jen Chen, Ming-Hsiung Hsieh, Yu-Chen Huang

26. Binge Alcohol Exposure Triggers Atrial Fibrillation Through T-Type $\mathrm{Ca}^{2+}$ Channel Upregulation via Protein Kinase C (PKC) / Glycogen Synthesis Kinase $3 \beta(G S K 3 \beta)$ / Nuclear Factor of Activated T-Cells (NFAT) Signaling - An Experimental Account of Holiday Heart Syndrome -

Yan Wang, Masaki Morishima, Dan Li, Naohiko Takahashi, Tetsunori Saikawa, Stanley Nattel, Katsushige Ono

27. Different Impact of Resting Heart Rate on Adverse Events in Paroxysmal and Sustained Atrial Fibrillation - The Fushimi AF Registry -

Moritake Iguchi, Yasuhiro Hamatani, Hiroaki Sugiyama, Kenjiro Ishigami, Yuya Aono, Syuhei Ikeda, Kosuke Doi, Akiko Fujino, Yoshimori An, Mitsuru Ishii, Nobutoyo Masunaga, Masahiro Esato, Hikari Tsuji, Hiromichi Wada, Koji Hasegawa, Hisashi Ogawa, Mitsuru Abe, Masaharu Akao on behalf of The Fushimi AF Registry Investigators unctional Role of the L396R Mutation of Tks5 Identified by an Exome-Wide Association Study in Atrial Fibrillation Xiaoxi Yang, Tetsuo Sasano, Yusuke Ebana, Jun K. Takeuchi, Kensuke Ihara, Masahiro Yamazoe, Tetsushi Furukawa 
29. Improved Risk Stratification of Patients With Brugada Syndrome by the New Japanese Circulation Society Guideline - A Multicenter Validation Study -

Akinori Wakamiya, Tsukasa Kamakura, Tetsuji Shinohara, Kenji Yodogawa, Nobuyuki Murakoshi, Hiroshi Morita, Naohiko Takahashi, Yasuya Inden, Wataru Shimizu, Akihiko Nogami, Minoru Horie, Kenzaburo Nakajima, Naoya Kataoka, Mitsuru Wada, Kenichiro Yamagata, Kohei Ishibashi, Yuko Inoue, Koji Miyamoto, Satoshi Nagase, Takashi Noda, Takeshi Aiba, Satoshi Yasuda, Masaki Ieda, Kengo Kusano

30. Novel Non-Invasive Index for Prediction of Responders in Cardiac Resynchronization Therapy Using HighResolution Magnetocardiography

Takahiro Nakashima, Shunsuke Usami, Takeshi Aiba, Shogo Oishi, Yoshitaka Kimura, Shoji Kawakami, Yuko Yamada-Inoue, Soshiro Ogata, Nobuhiko Ueda, Kenzaburo Nakajima, Tsukasa Kamakura, Mitsuru Wada, Kenichiro Yamagata, Kohei Ishibashi, Koji Miyamoto, Takashi Noda, Satoshi Nagase, Hideaki Kanzaki, Chisato Izumi, Satoshi Yasuda, Shiro Kamakura, Hiroshi Takaki, Masaru Sugimachi, Kengo Kusano

Cardiovascular Intervention (17)

31. Coronary Artery Perforation and Tamponade - Incidence, Risk Factors, Predictors and Outcomes From 12 Years' Data of the SCAAR Registry Jan Harnek, Stefan James, Bo Lagerqvist

32. Efficacy and Safety of Guideline-Recommended Risk Score-Directed Dual Antiplatelet Therapy After 2nd-Generation Drug-Eluting Stents

Ji-Yong Jang, Byoung-Kwon Lee, Jung-Sun Kim, Dong-Ho Shin, Sung-Jin Hong, Chul-Min Ahn, Byeong-Keuk Kim, Young-Guk Ko, Donghoon Choi, Myeong-Ki Hong, Kyung Woo Park, Hyeon-Cheol Gwon, Hyo-Soo Kim, Hyuck Moon Kwon, Yangsoo Jang

33. Comparison of Clinical Characteristics of Stent Thrombosis Between the Right Coronary Artery and the Left Coronary Artery - A Subanalysis of the REAL-ST Registry -

Yusuke Watanabe, Kenichi Sakakura, Hideo Fujita, Masanobu Ohya, Kazunori Horie, Futoshi Yamanaka, Gaku Nakazawa, Hiromasa Otake, Hiroki Shiomi, Masahiro Natsuaki, Kenji Ando, Kazushige Kadota, Shigeru Saito, Takeshi Kimura, Shoichi Kuramitsu on behalf of the REAL-ST Registry Investigators

34. Ventricular Fibrillation During Optical Coherence Tomography/Optical Frequency Domain Imaging - A Large Single-Center Experience -

Noriko Terada, Tatsuhiko Kuramochi, Tomoyo Sugiyama, Yoshihisa Kanaji, Masahiro Hoshino, Eisuke Usui, Masao Yamaguchi, Masahiro Hada, Toru Misawa, Yohei Sumino, Hidenori Hirano, Kai Nogami, Hiroki Ueno, Nobutaka Wakasa, Masahiro Hosokawa, Tadashi Murai, Tetsumin Lee, Taishi Yonetsu, Kazuhiko Kobashi, Tsunekazu Kakuta

35. Transradial vs. Transfemoral Percutaneous Coronary Intervention in Patients With or Without High Bleeding Risk Criteria

Ko Yamamoto, Masahiro Natsuaki, Takeshi Morimoto, Hiroki Shiomi, Hirotoshi Watanabe, Kyohei Yamaji, Hiroki Watanabe, Takao Kato, Naritatsu Saito, Kenji Ando, Kazushige Kadota, Yutaka Furukawa, Takeshi Kimura for the CREDO-Kyoto PCI/CABG Registry Cohort-2 Investigators

36. Long-Term Outcomes of Absorb Bioresorbable Vascular Scaffold vs. Everolimus-Eluting Metallic Stent - A Randomized Comparison Through 5 Years in Japan Ken Kozuma, Kengo Tanabe, Yuji Hamazaki, Takayuki Okamura, Jiro Ando, Yuji Ikari, Yoshihisa Nakagawa, Hajime Kusano, Divine Ediebah, Takeshi Kimura on behalf of the ABSORB Japan Investigators

37. Optical Coherence Tomography Comparison of Percutaneous Coronary Intervention Among Plaque Rupture, Erosion, and Calcified Nodule in Acute Myocardial Infarction

Amir Kh. M. Khalifa, Takashi Kubo, Yasushi Ino, Kosei Terada, Hiroki Emori, Daisuke Higashioka, Yosuke Katayama, Masahiro Takahata, Kunihiro Shimamura, Yasutsugu Shiono, Yoshiki Matsuo, Atsushi Tanaka, Takeshi Hozumi, Takashi Akasaka

38. Optical Coherence Tomography-Guided Percutaneous Coronary Intervention With Low-Molecular-Weight Dextran - Effect on Renal Function -

Kazumasa Kurogi, Masanobu Ishii, Kenji Sakamoto, Soichi Komaki, Hiroaki Kusaka, Nobuyasu Yamamoto, Seiji Takashio, Yuichiro Arima, Eiichiro Yamamoto, Koichi Kaikita, Kenichi Tsujita

39. Clopidogrel Monotherapy vs. Aspirin Monotherapy Following Short-Term Dual Antiplatelet Therapy in Patients Receiving Everolimus-Eluting Coronary Stent Implantation

Masahiro Natsuaki, Takeshi Morimoto, Hirotoshi Watanabe, Mitsuru Abe, Kazuya Kawai, Koichi Nakao, Kenji Ando, Kengo Tanabe, Yuji Ikari, Keiichi Igarashi Hanaoka, Yoshihiro Morino, Ken Kozuma, Kazushige Kadota, Takeshi Kimura on behalf of the STOPDAPT-1 and STOPDAPT-2 Trial Investigators

40. Comparison of Percutaneous Coronary Intervention, Coronary Artery Bypass Grafting and Medical Therapy in Non-ST Elevation Acute Coronary Syndrome Patients With 3-Vessel Disease

Sida Jia, Ce Zhang, Lin Jiang, Lianjun Xu, Jian Tian, Xueyan Zhao, Xinxing Feng, Dong Wang, Yin Zhang, Kai Sun, Jingjing Xu, Ru Liu, Bo Xu, Wei Zhao, Rutai Hui, Runlin Gao, Zhan Gao, Jinqing Yuan, Lei Song

41. Post-Procedural Anticoagulation After Primary Percutaneous Coronary Intervention for Anterior Acute Myocardial Infarction With Severe Left Ventricular Dysfunction

Peng-fei Chen, Jun-lin Yi, Jun-yu Pei, Liang Tang, Zhen-fei Fang, Sheng-hua Zhou, Xin-qun Hu 
42. Effects of Body Weight on Bleeding and Ischemic Events in Patients Undergoing Percutaneous Coronary Intervention - From the CREDO-Kyoto Registry Cohort-2 -

Ko Yamamoto, Masahiro Natsuaki, Yusuke Yoshikawa, Takeshi Morimoto, Hiroki Shiomi, Hirotoshi Watanabe, Takao Kato, Naritatsu Saito, Kazushige Kadota, Kenji Ando, Yutaka Furukawa, Toshihiro Tamura, Hiroki Sakamoto, Mamoru Toyofuku, Tsukasa Inada, Moriaki Inoko, Satoru Suwa, Tomoya Onodera, Katsuhisa Ishii, Norio Kanamori, Takeshi Kimura on behalf of the CREDO-Kyoto PCI/CABG Registry Cohort- Investigators

43. Clinical Outcomes and Angiographic Results of Bailout Stenting for Guide Catheter-Induced Iatrogenic Coronary Artery Dissection - Impact of Stent Type -

Hidewo Amano, Shunsuke Kubo, Kohei Osakada, Katsuya Miura, Masanobu Ohya, Takenobu Shimada, Ryosuke Murai, Takeshi Tada, Hiroyuki Tanaka, Yasushi Fuku, Tsuyoshi Goto, Kazushige Kadota

44. Predictors of Long-Term Infections After Cardiac Implantable Electronic Device Surgery - Utility of Novel PADIT and PACE DRAP Scores -

Sylwia Sławek-Szmyt, Aleksander Araszkiewicz, Marek Grygier, Krzysztof Szmyt, Lidia Chmielewska-Michalak, Wojciech Seniuk, Michał Waśniewski, Tomasz Smukowski, Maciej Lesiak, Przemysław Mitkowski

45. Vascular Response Occurring at 3 Months After Everolimus-Eluting Cobalt-Chromium Stent Implantation in Patients With ST-Segment Elevation Myocardial Infarction vs. Stable Coronary Artery Disease

Masaru Ishida, Daisuke Terashita, Tomonori Itoh, Hiromasa Otake, Yoshiro Tsukiyama, Tatsuo Kikuchi, Takatoshi Hayashi, Takahide Suzuki, Yoshiaki Ito, Takashi Morita, Kiyoshi Hibi, Takahiro Sawada, Takayuki Okamura, Junya Shite, Fumiaki Takahashi, Toshiro Shinke, Yoshihiro Morino

46. Long-Term Safety and Efficacy of Extended Dual Antiplatelet Therapy After Drug-Eluting Stent Implantation in Real-World Practice

Hye-Sung So, Min-Gyeong So, Seo-In Kang, Jong-Il Park, Jung-Hee Lee, Ung Kim, Jong-Seon Park

47. Implementation of Percutaneous Coronary Intervention During the COVID-19 Pandemic in Japan - Nationwide Survey Report of the Japanese Association of Cardiovascular Intervention and Therapeutics for Cardiovascular Disease Hideki Ishii, Tetsuya Amano, Kyohei Yamaji, Shun Kohsaka, Hiroyoshi Yokoi, Yuji Ikari

Cardiac Rehabilitation (4)

48. Pulsed Electromagnetic Fields Increase Angiogenesis and Improve Cardiac Function After Myocardial Ischemia in Mice Lihong Peng, Chenying Fu, Zejun Liang, Qing Zhang, Feng Xiong, Li Chen, Chengqi He, Quan Wei

49. Active Participation in Outpatient Cardiac Rehabilitation Is Associated With Better Prognosis After Coronary Artery Bypass Graft Surgery - J-REHAB CABG Study -

Hideki Origuchi, Haruki Itoh, Shin-ichi Momomura, Ryuji Nohara, Hiroyuki Daida, Takashi Masuda, Masahiro Kohzuki, Shigeru Makita, Kenji Ueshima, Masatoshi Nagayama, Kazuto Omiya, Hitoshi Adachi, Yoichi Goto

50. Hospital-Acquired Functional Decline and Clinical Outcomes in Older Patients Undergoing Transcatheter Aortic Valve Implantation

Masakazu Saitoh, Mike Saji, Aika Kozono-Ikeya, Takeshi Arimitsu, Akihiro Sakuyama, Hiromichi Ueki, Masatoshi Nagayama, Mitsuaki Isobe

51. Extended Sedentary Time Increases the Risk of All-Cause Death and New Cardiovascular Events in Patients With Diabetic Kidney Disease

Hajime Tamiya, Yuma Tamura, Syusuke Mochi, Yusuke Akazawa, Yumi Mochi, Nobuyuki Banba, Yuki Nakatani, Megumi Hoshiai, Asuka Ueno, Moeko Nagao, Takashi Tomoe, Masato Onozaki, Atsuko Uema, Atsuhiko Kawabe, Takushi Sugiyama, Takanori Yasu

Cardiovascular Surgery (9)

52. Incidence and Distribution of Cerebral Embolism After Cardiac Surgery According to the Systemic Perfusion Strategy - A Diffusion-Weighted Magnetic Resonance Imaging Study -

Hyung Gon Je, Min Ho Ju, Chee-Hoon Lee, Mi Hee Lim, Ji Hye Lee, Hye Rim Oh

53. Benefits of the Modified Bicaval Anastomosis Technique for Orthotopic Heart Transplantation From a SizeMismatched Marginal Donor

Takashi Kakuta, Satsuki Fukushima, Yusuke Shimahara, Shin Yajima, Naonori Kawamoto, Naoki Tadokoro, Norihide Fukushima, Soichiro Kitamura, Junjiro Kobayashi, Tomoyuki Fujita

54. Does Bilateral Internal Mammary Artery Grafting Better Suit Patients With Diabetes? - Two Different Ways to Explore Outcomes -

Chuan Wang, Ping Li, Fan Zhang, Qingyu Kong, Jingxing Li

55. Surgical Results for Infective Endocarditis Complicated With Cardiogenic Shock

Kazuma Handa, Daisuke Yoshioka, Koichi Toda, Jun-ya Yokoyama, Takaaki Samura, Kota Suzuki, Shigeru Miyagawa, Goro Matsumiya, Taichi Sakaguchi, Hirotsugu Fukuda, OSCAR Study Group, Yoshiki Sawa

56. Long-Term Outcomes of the Mosaic Aortic Porcine Bioprosthesis in Japan - Results From the Japan Mosaic Valve Long-Term Multicenter Study -

Yasushi Yoshikawa, Yukikatsu Okada, Yutaka Okita, Hitoshi Yaku, Junjiro Kobayashi, Hideyuki Uesugi, Shuichiro Takanashi, Toshiaki Ito, Tatsuya Nakao, Tadaaki Koyama, Taichi Sakaguchi, Kouji Yamamoto, Yoshiki Sawa

57. Surgical Aortic Valve Replacement for Aortic Stenosis in Dialysis Patients - Analysis of Japan Cardiovascular Surgery Database -

Takashi Yamauchi, Hiroyuki Yamamoto, Hiroaki Miyata, Junjiro Kobayashi, Takafumi Masai, Noboru Motomura

58. Impact of Mitral Valve Repair Case Volume on Postoperative Mortality - A Nationwide Korean Cohort Study Karam Nam, Eun Jin Jang, Jun Woo Jo, Jae Woong Choi, Jun Gi Jo, Jaehun Lee, Ho Geol Ryu 
59. Two-Year Results of a Multicenter Prospective Observational Study of the Zenith Spiral-Z Limb Deployed in the External Iliac Artery During Endovascular Aneurysm Repair

Naoki Fujimura, Tomohiro Imazuru, Hitoshi Matsumura, Tsuyoshi Shibata, Tadashi Furuyama, Kenjiro Kaneko, Hidetoshi Uchiyama, Noriyasu Morikage, Takayuki Uchida, Eiichi Teshima, Terutoshi Yamaoka, Hiroshi Masuhara, Hideki Ueda, Mamoru Arakawa, Togo Norimatsu, Hideaki Obara, Seiji Onitsuka

60. Bridge-to-Bridge Left Ventricular Assist Device Implantation Strategy vs. Primary Left Ventricular Assist Device Implantation Strategy

Teruhiko Imamura, Koichiro Kinugawa, Minoru Ono, Norihide Fukushima, Akira Shiose, Yoshiro Matsui, Kenji Yamazaki, Yoshikatsu Saiki, Goro Matsumiya, Hirokuni Arai, Yoshiki Sawa

Critical Care (6)

61. Osborn Wave Is Related to Ventricular Fibrillation and Tachycardia in Hypothermic Patients Nobunaga Okada, Tasuku Matsuyama, Sachiko Morita, Naoki Ehara, Nobuhiro Miyamae, Yohei Okada, Takaaki Jo, Yasuyuki Sumida, Makoto Watanabe, Masahiro Nozawa, Ayumu Tsuruoka, Yoshihiro Fujimoto, Yoshiki Okumura, Kunio Hamanaka, Tetsuhisa Kitamura, Kei Nishiyama, Bon Ohta

62. Heart Rate After Resuscitation From Out-of-Hospital Cardiac Arrest due to Acute Coronary Syndrome Is an Independent Predictor of Clinical Outcome

Shingo Matsumoto, Rine Nakanishi, Ippei Watanabe, Hiroto Aikawa, Ryota Noike, Takayuki Yabe, Ryo Okubo, Tadashi Fujino, Hideo Amano, Mikihito Toda, Takanori Ikeda

63. A Novel Extracorporeal Continuous-Flow Ventricular Assist System for Patients With Advanced Heart Failure - Initial Clinical Experience -

Osamu Seguchi, Tomoyuki Fujita, Nana Kitahata, Keiichiro Iwasaki, Kensuke Kuroda, Seiko Nakajima, Takuya Watanabe, Masanobu Yanase, Satsuki Fukushima, Tomonori Tsukiya, Nobumasa Katagiri, Toshihide Mizuno, Yoshiaki Takewa, Toshimitsu Hamasaki, Kaori Onda, Teruyuki Hayashi, Haruko Yamamoto, Eisuke Tatsumi, Junjiro Kobayashi, Norihide Fukushima

64. Prognostic Impact of No-Flow Time on 30-Day Neurological Outcomes in Patients With Out-of-Hospital Cardiac Arrest Who Received Extracorporeal Cardiopulmonary Resuscitation

Naoto Murakami, Nobuaki Kokubu, Nobutaka Nagano, Junichi Nishida, Ryo Nishikawa, Jun Nakata, Yohei Suzuki, Kazufumi Tsuchihashi, Eichi Narimatsu, Tetsuji Miura

65. Optimal Timing of Venoarterial-Extracorporeal Membrane Oxygenation in Acute Myocardial Infarction Patients Suffering From Refractory Cardiogenic Shock

Ki Hong Choi, Jeong Hoon Yang, David Hong, Taek Kyu Park, Joo Myung Lee, Young Bin Song, Joo-Yong Hahn, Seung-Hyuk Choi, Jin-Ho Choi, Su Ryeun Chung, Yang Hyun Cho, Dong Seop Jeong, Kiick Sung, Wook Sung Kim, Young Tak Lee, Hyeon-Cheol Gwon

66. Predictors of Survival to Discharge After Successful Weaning From Venoarterial Extracorporeal Membrane Oxygenation in Patients With Cardiogenic Shock

Donghoon Kim, Soo Jin Na, Yang Hyun Cho, Chi Ryang Chung, Kyeongman Jeon, Gee Young Suh, Taek Kyu Park, Joo Myung Lee, Young Bin Song, Joo-Yong Hahn, Jin-Ho Choi, Seung-Hyuk Choi, Hyeon-Cheol Gwon, Joong Hyun Ahn, Keumhee C. Carriere, Jeong Hoon Yang

Epidemiology (10)

67. Risk of Out-of-Hospital Cardiac Arrest in Aged Individuals in Relation to Cold Ambient Temperature - A Report From North Tochigi Experience -

Takashi Yoshinaga, Nobuyuki Shiba, Ryuji Kunitomo, Nobuyuki Hasegawa, Masanori Suzuki, Chuji Sekiguchi, Yotaro Shinozawa, Shunsuke Tsuge, Toshimitsu Kitajima, Yasuyuki Miyahara, Yoshio Misawa

68. Characteristics and Outcomes of Out-of-Hospital Cardiac Arrest in Educational Institutions in Japan - All-Japan Utstein Registry -

Chika Nishiyama, Kosuke Kiyohara, Tasuku Matsuyama, Tetsuhisa Kitamura, Takeyuki Kiguchi, Daisuke Kobayashi, Satoe Okabayashi, Tomonari Shimamoto, Takashi Kawamura, Taku Iwami

69. Association of Albuminuria With White Matter Hyperintensities Volume on Brain Magnetic Resonance Imaging in Elderly Japanese - The Hisayama Study -

Keisuke Yamasaki, Jun Hata, Yoshihiko Furuta, Naoki Hirabayashi, Tomoyuki Ohara, Daigo Yoshida, Yoichiro Hirakawa, Toshiaki Nakano, Takanari Kitazono, Toshiharu Ninomiya

70. Two-Year Recurrence After First-Ever Stroke in a General Population of 1.4 Million Japanese Patients - The Shiga Stroke and Heart Attack Registry Study -

Naoyuki Takashima, Hisatomi Arima, Yoshikuni Kita, Takako Fujii, Sachiko Tanaka-Mizuno, Satoshi Shitara, Akihiro Kitamura, Katsuyuki Miura, Kazuhiko Nozaki on behalf of the Shiga Stroke and Heart Attack Registry Group

71. Association Between Plasma Intact Parathyroid Hormone Levels and the Prevalence of Atrial Fibrillation in Patients With Chronic Kidney Disease - The Fukuoka Kidney Disease Registry Study -

Hokuto Arase, Shunsuke Yamada, Shigeru Tanaka, Masanori Tokumoto, Kazuhiko Tsuruya, Toshiaki Nakano, Takanari Kitazono

72. Impact of Cardiovascular Disease on Clinical Characteristics and Outcomes of Coronavirus Disease 2019 (COVID-19) Yangjing Xie, Qinghai You, Chaoran Wu, Shiyu Cao, Guangbo Qu, Xiaoxiang Yan, Xuan Han, Changhui Wang, Hong Zhang 
73. Clinical Differences of Recent Myocardial Infarction Compared With Acute Myocardial Infarction - Insights From the Tokyo CCU Network Multicenter Registry -

Ryosuke Ito, Jun Yamashita, Taishiro Chikamori, Seita Kondo, Yuya Mitsuhashi, Hiroshi Iwata, Mike Saji, Taku Asano, Kohei Wakabayashi, Kazuyuki Yahagi, Toshiro Shinke, Takaaki Mase, Kaito Abe, Hideki Miyachi, Satoshi Higuchi, Mikio Kishi, Hiroyuki Tanaka, Masao Yamasaki, Katsumi Miyauchi, Takeshi Yamamoto, Ken Nagao, Morimasa Takayama

74. Association of Cancer With Outcomes in Patients Hospitalized for Heart Failure Hidehiro Kaneko, Hidetaka Itoh, Haruki Yotsumoto, Hiroyuki Kiriyama, Tatsuya Kamon, Katsuhito Fujiu, Kojiro Morita, Nobuaki Michihata, Taisuke Jo, Norifumi Takeda, Hiroyuki Morita, Hideo Yasunaga, Issei Komuro

75. Serum Bilirubin in the Czech Population - Relationship to the Risk of Myocardial Infarction in Males Lenka Eremiasova, Jaroslav A Hubacek, Vilém Danzig, Věra Adamkova, Lenka Mrazova, Jan Pitha, Věra Lanska, Renata Cífková, Libor Vitek

76. Current Trends in Epidemiology and Clinical Features of Thromboangiitis Obliterans in Japan - A Nationwide Survey Using the Medical Support System Database -

Yoshiko Watanabe, Tetsuro Miyata, Kunihiro Shigematsu, Kazuo Tanemoto, Yoshikazu Nakaoka, Masayoshi Harigai, Japan Research Committee of the Ministry of Health, Labour, and Welfare for Intractable Vasculitis (JPVAS)

Heart Failure (25)

77. Relationship Between Respiratory Compensation Point and Anaerobic Threshold in Patients With Heart Failure With Reduced Ejection Fraction

Taisuke Nakade, Hitoshi Adachi, Makoto Murata, Shigeto Naito

78. Effects of Angiotensin-Converting Enzyme Inhibitors and Angiotensin-Receptor Blockers in Heart Failure With Chronic Kidney Disease - Propensity Score Matching Analysis -

Hyun-Jin Kim, Min-Ho Lee, Sang-Ho Jo, Won-Woo Seo, Sung Eun Kim, Kyung-Jin Kim, Jin-Oh Choi, Hyo-Suk Ahn, Dong-Ju Choi, Kyu-Hyung Ryu

79. Incidence and Clinical Significance of 30-Day and 90-Day Rehospitalization for Heart Failure Among Patients With Acute Decompensated Heart Failure in Japan - From the NARA-HF Study -

Satomi Ishihara, Rika Kawakami, Maki Nogi, Kaeko Hirai, Yukihiro Hashimoto, Yasuki Nakada, Hitoshi Nakagawa, Tomoya Ueda, Taku Nishida, Kenji Onoue, Tsunenari Soeda, Satoshi Okayama, Makoto Watanabe, Yoshihiko Saito

80. Association of Local Epicardial Adipose Tissue Depots and Left Ventricular Diastolic Performance in Patients With Preserved Left Ventricular Ejection Fraction

Gulinu Maimaituxun, Hirotsugu Yamada, Daiju Fukuda, Shusuke Yagi, Kenya Kusunose, Yukina Hirata, Susumu Nishio, Takeshi Soeki, Hiroaki Masuzaki, Masataka Sata, Michio Shimabukuro

81. Lifestyle Modification or Medication to Improve Condition of Patients With Asymptomatic Heart Failure - Monitoring and Management of OptiVol Alert to Reduce Heart Failure Hospitalization II (MOMOTARO II) Study -

Akihito Miyoshi, Nobuhiro Nishii, Yoji Okamoto, Shinpei Fujita, Kenji Kawamoto, Keisuke Okawa, Shigeki Hiramatsu, Kazufumi Nakamura, Hiroshi Morita, Hiroshi Ito

82. Development and Practical Test of Quality Indicators for Palliative Care in Patients With Chronic Heart Failure Yasuhiro Hamatani, Yasuko Takada, Yoshihiro Miyamoto, Yukie Kawano, Yuta Anchi, Tatsuhiro Shibata, Atsushi Suzuki, Mitsunori Nishikawa, Hiroto Ito, PhD, Masashi Kato, Tsuyoshi Shiga, Yoshihiro Fukumoto, Chisato Izumi, Satoshi Yasuda, Hisao Ogawa, Yasuo Sugano, Toshihisa Anzai

83. Systemic Inflammatory Response Syndrome Is a Major Determinant of Cardiovascular Outcome in Takotsubo Syndrome Lucie Lachmet-Thébaud, Benjamin Marchandot, Kensuke Matsushita, Charlotte Dagrenat, Marilou Peillex, Chisato Sato, Antonin Trimaille, Antje Reydel, Annie Trinh, Patrick Ohlmann, Laurence Jesel, Olivier Morel

84. Impact of Hospital Practice Factors on Mortality in Patients Hospitalized for Heart Failure in Japan - An Analysis of a Large Number of Health Records From a Nationwide Claims-Based Database, the JROAD-DPC -

Hidetaka Kaku, Kouta Funakoshi, Tomomi Ide, Takeo Fujino, Shouji Matsushima, Kisho Ohtani, Taiki Higo, Michikazu Nakai, Yoko Sumita, Kunihiro Nishimura, Yoshihiro Miyamoto, Toshihisa Anzai, Hiroyuki Tsutsui

85. Effect of the Creatinine Excretion Rate Index, a Marker of Sarcopenia, on Prediction of Intracranial Hemorrhage in

Patients With Advanced Heart Failure and a Continuous-Flow Left Ventricular Assist Device

Keiichiro Iwasaki, Osamu Seguchi, Shunsuke Murata, Kunihiro Nishimura, Koichi Yoshitake, Nobuichiro Yagi, Yasumori Sujino, Eiji Anegawa, Hiroki Mochizuki, Kensuke Kuroda, Seiko Nakajima, Takuya Watanabe, Masanobu Yanase, Satsuki Fukushima, Tomoyuki Fujita, Junjiro Kobayashi, Hiroshi Ito, Norihide Fukushima

86. Efficacy of Rapid Decongestion Strategy in Patients Hospitalized for Acute Heart Failure

Mitsutoshi Oguri, Hideki Ishii, Kunihiko Takahara, Kenichiro Yasuda, Tomonobu Takikawa, Takuya Sumi, Hiroshi Takahashi, Toyoaki Murohara

Effect of Therapeutic Modification on Outcomes in Heart Transplantation Over the Past Two Decades - A SingleCenter Experience in Japan -

Masanobu Yanase, Keiichiro Iwasaki, Takuya Watanabe, Osamu Seguchi, Seiko Nakajima, Kensuke Kuroda, Hiroki Mochizuki, Sachi Matsuda, Hiromi Takenaka, Megumi Ikura, Naoki Tadokoro, Satsuki Fukushima, Tomoyuki Fujita, Hatsue Ishibashi-Ueda, Takeshi Nakatani, Soichiro Kitamura, Junjiro Kobayashi, Kenichi Tsujita, Hisao Ogawa, Norihide Fukushima 
88. Non-Invasive Central Venous Pressure Measurement Using Enclosed-Zone Central Venous Pressure (ezCVPTM)

Takayuki Hidaka, Yoji Sumimoto, Yoshihiro Dohi, Haruka Morimoto, Hitoshi Susawa, Kazuhiro Nitta, Ken Ishibashi, Satoru Kurisu, Yukihiro Fukuda, Haruki Hashimoto, Shogo Matsui, Shinji Kishimoto, Masato Kajikawa, Tatsuya Maruhashi, Teiji Ukawa, Chikara Goto, Ayumu Nakashima, Kensuke Noma, Toshio Tsuji, Yasuki Kihara, Yukihito Higashi

89. Evaluation of an Integrated Device Diagnostics Algorithm to Risk Stratify Heart Failure Patients - Results From the SCAN-HF Study -

Ken Okumura, Shingo Sasaki, Kengo Kusano, Takanao Mine, Kenshi Fujii, Atsushi Iwasa, Osahiko Sunagawa, Hiroshige Yamabe, Naohiko Takahashi, Shunsuke Ishii, Yasuchika Takeishi, Naoya Tsuboi, Satoshi Shizuta, Kazutaka Aonuma, Akira Shimane, Hiroshi Tada, Toshiyuki Ishikawa, Ryusuke Tsunoda, Tetsuya Numata, Yasushi Mukai, Yasuki Kihara, Jodi Koehler, Kazuhiro Hidaka, Vinod Sharma

90. Recovery From Exhaustion of the Frank-Starling Mechanism by Mechanical Unloading With a Continuous-Flow Ventricular Assist Device

Shunsuke Saito, Koichi Toda, Shigeru Miyagawa, Yasushi Yoshikawa, Hiroki Hata, Daisuke Yoshioka, Fusako Sera, Kei Nakamoto, Takashi Daimon, Yasushi Sakata, Yoshiki Sawa

91. Prognosis and Clinical Characteristics of Dilated Cardiomyopathy With Family History via Pedigree Analysis Kyohei Marume, Teruo Noguchi, Emi Tateishi, Yoshiaki Morita, Hiroyuki Miura, Kunihiro Nishimura, Keiko Ohta-Ogo, Naoaki Yamada, Kenichi Tsujita, Chisato Izumi, Kengo Kusano, Hisao Ogawa, Satoshi Yasuda

92. Poor Increase in Pulse Pressure During Cardiopulmonary Exercise Testing Predicts Cardiovascular Death of Patients With Heart Failure With Reduced Ejection Fraction

Taisuke Nakade, Hitoshi Adachi, Makoto Murata, Shigeto Naito

93. Clinical Characteristics and Outcomes of Heart Failure Patients With Long-Term Care Insurance - Insights From the Kitakawachi Clinical Background and Outcome of Heart Failure Registry -

Kensuke Takabayashi, Kotaro Iwatsu, Tsutomu Ikeda, Yuko Morikami, Tahei Ichinohe, Takashi Yamamoto, Kotoe Takenaka, Hiroyuki Takenaka, Hiroyuki Muranaka, Ryoko Fujita, Miyuki Okuda, Osamu Nakajima, Hitoshi Koito, Yuka Terasaki, Tetsuhisa Kitamura, Shouji Kitaguchi, Ryuji Nohara

94. Efficacy of Pulmonary Artery Pulsatility Index as a Measure of Right Ventricular Dysfunction in Stable Phase of Dilated Cardiomyopathy

Tasuku Kuwayama, Ryota Morimoto, Hideo Oishi, Hiroo Kato, Yuki Kimura, Shingo Kazama, Naoki Shibata, Yoshihito Arao, Shogo Yamaguchi, Hiroaki Hiraiwa, Toru Kondo, Kenji Furusawa, Takahiro Okumura, Toyoaki Murohara

95. Differential Impact of the Renal Resistive Index on Future Cardiovascular Events in Hospitalized Atherosclerotic Cardiovascular Patients According to Left Ventricular Ejection Fraction - The Jichi Vascular Hemodynamics in Hospitalized Cardiovascular Patients (J-VAS) Study -

Praew Kotruchin, Satoshi Hoshide, Hiromi Ueno, Hayato Shimizu, Takahiro Komori, Kazuomi Kario

96. Impact of Prior Ischemic Stroke on Outcomes in Patients With Heart Failure - A Propensity-Matched Study Yu Wang, Meng-Xi Yang, Qiang Tu, Li-Yuan Tao, Gang Liu, Hui An, Hu Zhang, Jiang-Li Jin, Jia-Sai Fan, Yi-Fei Du, Jin-Gang Zheng, Jing-Yi Ren

97. Dapagliflozin Influences Ventricular Hemodynamics and Exercise-Induced Pulmonary Hypertension in Type 2 Diabetes Patients - A Randomized Controlled Trial -

Hiroyuki Kayano, Shinji Koba, Tsutomu Hirano, Taiju Matsui, Hiroto Fukuoka, Hiroaki Tsuijita, Shigeto Tsukamoto, Toshiyuki Hayashi, Tsutomu Toshida, Norikazu Watanabe, Yuji Hamazaki, Eiichi Geshi, Mikitaka Murakami, Kazuo Aihara, Koujin Kaneko, Hirokazu Yamada, Youichi Kobayashi, Toshiro Shinke

98. Incidence, Factors, and Prognostic Impact of Re-Exploration for Bleeding After Continuous-Flow Left Ventricular Assist Device Implantation - A Japanese Single-Center Study -

Keiichiro Iwasaki, Koichi Yoshitake, Nobuichiro Yagi, Yasumori Sujino, Eiji Anegawa, Hiroki Mochizuki, Kensuke Kuroda, Seiko Nakajima, Takuya Watanabe, Osamu Seguchi, Masanobu Yanase, Satsuki Fukushima, Tomoyuki Fujita, Junjiro Kobayashi, Hiroshi Ito, Norihide Fukushima

99. High Prevalence of Left Ventricular Non-Compaction and Its Effect on Chemotherapy-Related Cardiac Dysfunction in Patients With Hematological Diseases

Mitsuhito Hirano,Koichi Kimura, Tomohiro Ishigaki, Masanori Nojima, Masao Daimon, Hiroyuki Morita, Katsu Takenaka, Boqing Xu, Naoko Sawada, Megumi Hirokawa, Issei Komuro, Takayuki Morisaki, Hiroshi Yotsuyanagi, Toyotaka Kawamata, Kazuaki Yokoyama, Takaaki Konuma, Seiko Kato, Hiroshi Yasui, Tokiko Nagamura-Inoue, Kaoru Uchimaru, Satoshi Takahashi, Yoichi Imai, Arinobu Tojo

100. Influence of Induction Therapy Using Basiliximab With Delayed Tacrolimus Administration in Heart Transplant Recipients - Comparison With Standard Tacrolimus-Based Triple Immunosuppression -

Takuya Watanabe, Masanobu Yanase, Osamu Seguchi, Tomoyuki Fujita, Toshimitsu Hamasaki, Seiko Nakajima, Kensuke Kuroda, Yuto Kumai, Koichi Toda, Keiichiro Iwasaki, Yuki Kimura, Hiroki Mochizuki, Eiji Anegawa, Yasumori Sujino, Nobuichiro Yagi, Koichi Yoshitake, Kyoichi Wada, Sachi Matsuda, Hiromi Takenaka, Megumi Ikura, Kazuki Nakagita, Shin Yajima, Yorihiko Matsumoto, Naoki Tadokoro, Takashi Kakuta, Satsuki Fukushima, Hatsue Ishibashi-Ueda, Junjiro Kobayashi, Norihide Fukushima 
101. Clinical Risk Factors and Prognostic Impact of Osteoporosis in Patients With Chronic Heart Failure

Satoshi Katano, Toshiyuki Yano, Takanori Tsukada, Hidemichi Kouzu, Suguru Honma, Takuya Inoue, Yuhei Takamura, Ryohei Nagaoka, Tomoyuki Ishigo, Ayako Watanabe, Katsuhiko Ohori, Masayuki Koyama, Nobutaka Nagano, Takefumi Fujito, Ryo Nishikawa, Hiroyuki Takashima, Akiyoshi Hashimoto, Masaki Katayose, Tetsuji Miura

Hypertension and Circulatory Control (1)

102. Training-Induced Deactivation of the AT 1 Receptor Pathway Drives Autonomic Control and Heart Remodeling

During the Transition From the Pre- to Hypertensive Phase in Spontaneously Hypertensive Rats

Tassia Santos Rodrigues da Costa, Gustavo Santos Masson, Rosangela Aparecida dos Santos Eichler, Juliane Cristina de Souza Silva, Silvia Lacchini, Lisete Compagno Michelini

Imaging (11)

103. Efficacy and Reproducibility of Attenuation-Compensated Optical Coherence Tomography for Assessing External Elastic Membrane Border and Plaque Composition in Native and Stented Segments - An In Vivo and HistologyBased Study -

Anantharaman Ramasamy, Jaryl Ng, Stephen White, Thomas W. Johnson, Nicolas Foin, Michael J.A. Girard, Jouke Dijkstra, Rajiv Amersey, Simon Scoltock, Sudheer Koganti, Daniel Jones, Chongying Jin, Lorenz Räber, Patrick W. Serruys, Ryo Torii, Tom Crake, Roby Rakhit, Andreas Baumbach, Anthony Mathur, Christos V. Bourantas

104. Japanese Survey of Radiation Dose Associated With Coronary Computed Tomography Angiography - 2013 Data From a Multicenter Registry in Daily Practice -

Yuki Tanabe, Teruhito Kido,Fumiko Kimura, Yasuyuki Kobayashi, Naofumi Matsunaga, Kunihiro Yoshioka, Norihiko Yoshimura, Teruhito Mochizuki

105. Validation of Intraoperative Catheter Phase Mapping Using a Simultaneous Optical Measurement System in Rabbit Ventricular Myocardium

Naoki Tomii, Keisuke Asano, Hiroshi Seno, Takashi Ashihara, Ichiro Sakuma, Masatoshi Yamazaki

106. Extent of Late Gadolinium Enhancement Predicts Thromboembolic Events in Patients With Hypertrophic Cardiomyopathy

Anna Hohneck, Daniel Overhoff, Christina Doesch, Raphael Sandberg, Boris Rudic, Erol Tueluemen, Johannes Budjan, Kristina Szabo, Martin Borggrefe, Theano Papavassiliu

107. Magnetic Resonance Imaging Evaluation of Left Common Iliac Vein Compression in Patients With and Without Symptoms of Venous Disease

Larissa Maria Giacon Costa, Adriano Tachibana, Fernanda da Silva Magao, Nelson Wolosker, Ronaldo Hueb Baroni

108. Doppler-Derived Intrarenal Venous Flow Mirrors Right-Sided Heart Hemodynamics in Patients With Cardiovascular Disease Yoshihiro Seo, Noriko Iida, Masayoshi Yamamoto, Tomoko Ishizu, Masaki Ieda, Nobuyuki Ohte

109. Hybrid Assessment of Myocardial Ischemia Using Stress-Only Nuclear Myocardial Perfusion Imaging and Rest Computed Tomography Perfusion Imaging

Yasuyuki Suzuki, Naoya Matsumoto, Ayano Makita, Tadashi Ashida, Keiichiro Kuronuma, Yasuo Amano, Shunichi Yoda, Yasuo Okumura

110. Concomitant Use of Rosuvastatin and Eicosapentaenoic Acid Significantly Prevents Native Coronary Atherosclerotic Progression in Patients With In-Stent Neoatherosclerosis

Yoichiro Sugizaki, Hiromasa Otake, Koji Kuroda, Hiroyuki Kawamori, Takayoshi Toba, Akira Nagasawa, Ryo Takeshige, Shinsuke Nakano, Yoichiro Matsuoka, Kosuke Tanimura, Yu Takahashi, Yusuke Fukuyama, Ken-ichi Hirata

111. Prognostic Significance of a Combination of QRS Score and E/e' Obtained 2 Weeks After the Onset of ST-Elevation Myocardial Infarction

Noriaki Iwahashi, Masaomi Gohbara, Jin Kirigaya, Takeru Abe, Mutsuo Horii, Hironori Takahashi, Masami Kosuge, Yohei Hanajima, Eiichi Akiyama, Kozo Okada, Yasushi Matsuzawa, Nobuhiko Maejima, Kiyoshi Hibi, Toshiaki Ebina, Kouichi Tamura, Kazuo Kimura

112. In Vivo Evaluation of Tissue Protrusion by Using Optical Coherence Tomography and Coronary Angioscopy Immediately After Stent Implantation

Shunsuke Sakai, Akira Sato, Tomoya Hoshi, Daigo Hiraya, Hiroaki Watabe, Masaki Ieda

113. Impact of Neointimal Condition and Platelet Reactivity on Intrastent Thrombus at Long-Term Follow-up After 2nd- and 3rd-Generation Drug-Eluting Stent Implantation - Insights From a Coronary Angioscopy and Pharmacodynamic Study -

Takero Matsuura, Masafumi Ueno, Heitaro Watanabe, Masakazu Yasuda, Toru Takase, Takashi Nakamura, Kenji Yamaji, Yoshitaka Iwanaga, Shunichi Miyazaki, Gaku Nakazawa

Ischemic Heart Disease (21)

114. Prasugrel for Japanese Patients With Ischemic Heart Disease in Long-Term Clinical Practice (PRASFIT-Practice II) - 1-Year Follow-up Results of a Postmarketing Observational Study -

Masato Nakamura, Takanari Kitazono, Ken Kozuma, Toru Sekine, Shinya Nakamura, Kazuhito Shiosakai, Tomoko Iizuka 
115. Influence of Preadmission Frailty on Short- and Mid-Term Prognoses in Octogenarians With ST-Elevation Myocardial Infarction

Naoki Yoshioka, Kensuke Takagi, Itsuro Morishima, Yasuhiro Morita, Yusuke Uemura, Yosuke Inoue, Norio Umemoto, Naoki Shibata, Yosuke Negishi, Ruka Yoshida, Akihito Tanaka, Hideki Ishii, Toyoaki Murohara on behalf of the N-Registry Investigators

116. Age- and Gender-Related Differences in Coronary Lesion Plaque Composition on Optical Coherence Tomography Toshimitsu Sato, Yoshiyasu Minami, Kiyoshi Asakura, Masahiro Katamine, Ayami Kato, Aritomo Katsura, Yusuke Muramatsu, Ryota Kakizaki, Teruyoshi Nemoto, Takuya Hashimoto, Kazuhiro Fujiyoshi, Ryo Kameda, Kentaro Meguro, Takao Shimohama, Junya Ako

117. Diabetes Mellitus and Long-Term Risk for Heart Failure After Coronary Revascularization Yasuaki Takeji, Hiroki Shiomi, Takeshi Morimoto, Yutaka Furukawa, Natsuhiko Ehara, Yoshihisa Nakagawa, Takao Kato, Junichi Tazaki, Eri Toda Kato, Hidenori Yaku, Yusuke Yoshikawa, Tomohisa Tada, Michiya Hanyu, Kazushige Kadota, Tatsuhiko Komiya, Kenji Ando, Takeshi Kimura, CREDO-Kyoto PCI/CABG Registry Cohort Investigators

118. East Asians Variant Mitochondrial Aldehyde Dehydrogenase 2 Genotype Exacerbates Nitrate Tolerance in Patients With Coronary Spastic Angina

Yuji Mizuno, Eisaku Harada, Fumihito Kugimiya, Makoto Shono, Izumi Kusumegi, Michihiro Yoshimura, Kenji Kinoshita, Hirofumi Yasue

119. DNA Hypomethylation of miR-30a Mediated the Protection of Hypoxia Postconditioning Against Aged Cardiomyocytes Hypoxia/Reoxygenation Injury Through Inhibiting Autophagy

YanHua Wang, YinJu Hao, Hui Zhang, LingBo Xu, Ning Ding, Rui Wang, GuangRong Zhu, ShengChao Ma, AnNing Yang, Yong Yang, Kai Wu, YuanXu Jiang, HuiPing Zhang, YiDeng Jiang

120. Atherosclerotic Coronary Plaque Is Associated With Adventitial Vasa Vasorum and Local Inflammation in Adjacent Epicardial Adipose Tissue in Fresh Cadavers

Hiroyuki Ito, Tetsuzo Wakatsuki, Koji Yamaguchi, Daiju Fukuda, Yutaka Kawabata, Tomomi Matsuura, Kenya Kusunose, Takayuki Ise, Takeshi Tobiume, Shusuke Yagi, Hirotsugu Yamada, Takeshi Soeki, Yoshihiro Tsuruo, Masataka Sata

121. Platelet-Derived Thrombogenicity Measured by Total Thrombus-Formation Analysis System in Patients With STSegment Elevation Myocardial Infarction Undergoing Primary Percutaneous Coronary Intervention Shinnosuke Kikuchi, Kengo Tsukahara, Shinya Ichikawa, Takeru Abe, Yugo Minamimoto, Yuichiro Kimura, Eiichi Akiyama, Naoki Nakayama, Kozo Okada, Yasushi Matsuzawa, Masaaki Konishi, Nobuhiko Maejima, Noriaki Iwahashi, Kiyoshi Hibi, Masami Kosuge, Toshiaki Ebina, Kouichi Tamura, Kazuo Kimura

122. Proteomics Profiling Reveals Insulin-Like Growth Factor 1, Collagen Type VI $\alpha$-2 Chain, and Fermitin Family Homolog 3 as Potential Biomarkers of Plaque Erosion in ST-Segment Elevated Myocardial Infarction Jinxin Liu, Shanjie Wang, Jingbo Hou, Hengxuan Cai, Weili Pan, Haimeng Dong, Rong Sun, Hui Dong, Shaohong Fang, Bo Yu

123. Apolipoprotein B/A-I Ratio Predicts Lesion Severity and Clinical Outcomes in Diabetic Patients With Acute Coronary Syndrome

Yue Liu, Si-da Jia, De-shan Yuan, Na Xu, Lin Jiang, Zhan Gao, Jue Chen, Yue-jin Yang, Run-lin Gao, Bo Xu, Jin-qing Yuan

124. In-Hospital Mortality in Acute Myocardial Infarction According to Population Density and Primary Angioplasty Procedures Volume

Yasushi Matsuzawa, Masaaki Konishi, Michikazu Nakai, Yusuke Saigusa, Masataka Taguri, Masaomi Gohbara, Toshiaki Ebina, Masami Kosuge, Kiyoshi Hibi, Kunihiro Nishimura, Yoshihiro Miyamoto, Satoshi Yasuda, Hisao Ogawa, Yoshihiko Saito, Naoki Nakayama, Ichiro Takeuchi, Kouichi Tamura, Kazuo Kimura

125. All Resting Physiological Indices May Not Be Equivalent - Comparison Between the Diastolic Pressure Ratio and Resting Full-Cycle Ratio -

Masahiro Hoshino, Taishi Yonetsu, Tomoyo Sugiyama, Yoshihisa Kanaji, Rikuta Hamaya, Yoshinori Kanno, Masahiro Hada, Masao Yamaguchi, Yohei Sumino, Eisuke Usui, Hidenori Hirano, Tomoki Horie, Kai Nogami, Hiroki Ueno, Toru Misawa, Tadashi Murai, Tetsumin Lee, Tsunekazu Kakuta

126. Bone Marrow Mesenchymal Stem Cell-Derived Exosomal miRNA-29c Decreases Cardiac Ischemia/Reperfusion Injury Through Inhibition of Excessive Autophagy via the PTEN/Akt/mTOR Signaling Pathway

Te Li, Junlian Gu, Ou Yang, Jianmeng Wang, Yonggang Wang, Jian Kong

127. Prevalence of the Academic Research Consortium for High Bleeding Risk Criteria and Prognostic Value of a Simplified Definition

Katsuya Miura, Takenobu Shimada, Masanobu Ohya, Ryosuke Murai, Hidewo Amano, Shunsuke Kubo, Takeshi Tada, Hiroyuki Tanaka, Yasushi Fuku, Tsuyoshi Goto, Kazushige Kadota

128. Independent Factors for In-Hospital Death Following Drug-Eluting Stent Thrombosis From the Japanese Adverse Event Report System

Yoshiaki Mitsutake, Akihide Konishi, Nobuhiro Handa, Mami Ho, Haruki Shirato, Takuya Ito, Kazuhisa Koike, Shuichi Mochizuki, Kensuke Ishii 
129. Comparison Between Clopidogrel and Prasugrel Associated With CYP2C19 Genotypes in Patients Receiving Percutaneous Coronary Intervention in a Japanese Population

Yuichi Sawayama, Takashi Yamamoto, Yukinori Tomita, Kohei Asada, Noriaki Yagi, Megumi Fukuyama, Akashi Miyamoto, Hiroshi Sakai, Tomoya Ozawa, Tetsuichiro Isono, Daiki Hira, Tomohiro Terada, Minoru Horie, Yoshihisa Nakagawa

130. Incidence of Cardiovascular Events and Safety Profile of Prasugrel in Korean Patients With Acute Coronary Syndrome

Min-Ku Chon, Soon-Myung Jung, Soo-Yong Lee, Sang-Hyun Lee, Ki-Won Hwang, Jin-Hee Choi, Jeong-Su Kim, Yong-Hyun Park, June-Hong Kim, Kook-Jin Chun

131. Increased Serum Malondialdehyde-Modified Low-Density Lipoprotein and Coronary Angiographic Progression After Drug-Eluting Stent Implantation in Patients With Stable Angina

Masashi Yokoi, Tsuyoshi Ito, Hiroshi Fujita, Tomonori Sugiura, Yoshihiro Seo, Nobuyuki Ohte

132. A Practical Risk Score to Predict 24-Month Post-Discharge Mortality Risk in Patients With Non-ST-Segment Elevation Myocardial Infarction

Rui Fu, Chenxi Song, Jingang Yang, Chuanyu Gao, Yan Wang, Haiyan Xu, Xiaojin Gao, Xiaoxue Fan, Han Xu, Hao Wang, Kefei Dou, Yuejin Yang on behalf of the CAMI Registry Study

133. Prasugrel for Japanese Patients With Ischemic Heart Disease in Long-Term Clinical Practice (PRASFIT-Practice II)

- Final 2-Year Follow-up Results of a Postmarketing Observational Study -

Masato Nakamura, Takanari Kitazono, Ken Kozuma, Toru Sekine, Shinya Nakamura, Kazuhito Shiosakai, Ayumi Tanabe, Tomoko Iizuka

134. Comparison of Optical Flow Ratio and Fractional Flow Ratio in Stent-Treated Arteries Immediately After Percutaneous Coronary Intervention

Hiroki Emori, Takashi Kubo, Yasutsugu Shiono, Yasushi Ino, Kunihiro Shimamura, Kosei Terada, Takahiro Nishi, Daisuke Higashioka, Masahiro Takahata, Teruaki Wada, Manabu Kashiwagi, Amir Kh. M. Khalifa, Atsushi Tanaka, Takeshi Hozumi, Shengxian Tu, Takashi Akasaka

olic Disorder (2)

135. Midkine Inhibits Cholesterol Efflux by Decreasing ATP-Binding Membrane Cassette Transport Protein A1 via Adenosine Monophosphate-Activated Protein Kinase/Mammalian Target of Rapamycin Signaling in Macrophages Han-xiao Ou, Qin Huang, Chu-hao Liu, Ji Xiao, Yun-cheng Lv, Xuan Li, Li-Ping Lei, Zhong-cheng Mo

136. Short-Term Efficacy (at 12 Weeks) and Long-Term Safety (up to 52 Weeks) of Omega-3 Free Fatty Acids (AZD0585) for the Treatment of Japanese Patients With Dyslipidemia - A Randomized, Double-Blind, Placebo-Controlled, Phase III Study -

Koutaro Yokote, Kiyoshi Niwa, Tomomi Hakoda, Fumiki Oh, Yoshitaka Kajimoto, Toshiki Fukui, Hyosung Kim, Yoshinori Noda, Torbjörn Lundström, Toshitaka Yajima

Molecular Cardiology (2)

137. M2b Macrophages Regulate Cardiac Fibroblast Activation and Alleviate Cardiac Fibrosis After Reperfusion Injury Yuan Yue, Suiqing Huang, Lexun Wang, Zixuan Wu, Mengya Liang, Huayang Li, Linhua Lv, Wei Li, Zhongkai Wu

138. Hsa-circ_0010283 Regulates Oxidized Low-Density Lipoprotein-Induced Proliferation and Migration of Vascular Smooth Muscle Cells by Targeting the miR-133a-3p/Pregnancy-Associated Plasma Protein A Axis

Zibo Feng, Youpeng Zhu, Jing Zhang, Wenbo Yang, Zhimin Chen, Binghui Li

Myocardial Disease (4)

139. Co-Phenotype of Left Ventricular Non-Compaction Cardiomyopathy and Atypical Catecholaminergic Polymorphic Ventricular Tachycardia in Association With R169Q, a Ryanodine Receptor Type 2 Missense Mutation Yoshihiro Nozaki, Yoshiaki Kato, Kiyoshi Uike, Kenichiro Yamamura, Masahiro Kikuchi, Maki Yasuda, Seiko Ohno, Minoru Horie, Takashi Murayama, Nagomi Kurebayashi, Hitoshi Horigome

140. New Appearance of Fragmented QRS as a Predictor of Ventricular Arrhythmic Events in Patients With Hypertrophic Cardiomyopathy

Soichiro Ogura, Kazufumi Nakamura, Hiroshi Morita, Norihisa Toh, Koji Nakagawa, Masashi Yoshida, Atsuyuki Watanabe, Nobuhiro Nishii, Toru Miyoshi, Hiroshi Ito

141. Sustaining Circulating Regulatory T Cell Subset Contributes to the Therapeutic Effect of Paroxetine on Mice With Diabetic Cardiomyopathy

Yongsheng Han, Jiacheng Lai, Juan Tao, Yu Tai, Weijie Zhou, Paipai Guo, Zhen Wang, Manman Wang, Qingtong Wang

142. Lifelong Clinical Impact of the Presence of Sarcomere Gene Mutation in Japanese Patients With Hypertrophic Cardiomyopathy

Yasuteru Nakashima, Toru Kubo, Kenta Sugiura, Yuri Ochi, Asa Takahashi, Yuichi Baba, Takayoshi Hirota, Naohito Yamasaki, Akinori Kimura, Yoshinori L. Doi, Hiroaki Kitaoka

Pediatric Cardiology and Adult Congenital Heart Disease (8)

143. Simultaneous Volumetric and Functional Assessment of the Right Ventricle in Hypoplastic Left Heart Syndrome After Fontan Palliation, Utilizing 3-Dimensional Speckle-Tracking Echocardiography Tomoyuki Sato, Renzo JC Calderon, Berthold Klas, Gianni Pedrizzetti, Anirban Banerjee 
144. The Impact of Post-Graduate Year of Primary Surgeon on Technical Performance Score in Tetralogy of Fallot Repair Masatoshi Shimada, Takaya Hoashi, Jun Iida, Hajime Ichikawa

145. Novel Strategy for Predicting Conduction Abnormalities During Transcatheter Closure of Perimembranous Ventricular Septal Defect in Adults

Lihong Tang, Xianzhang Zhan, Caojin Zhang, Xianhong Fang, Hongtao Liao, Fangzhou Liu, Weidong Lin, Yigao Huang, Tao Huang, Hongwen Fei, Shulin Wu, Yumei Xue

146. Partnership Between Japan and the United States for Early Development of Pediatric Medical Devices - Harmonization By Doing for Children -

Sara Takahashi, Nicole Ibrahim, Satoshi Yasukochi, Richard Ringel, Frank Ing, Hideshi Tomita, Hisashi Sugiyama, Masaaki Yamagishi, Thomas J. Forbes, Sung-Hae Kim, Mami Ho, Nicole Gillette, Yasuko Nakamura, Koji Mineta, Neal Fearnot, Declan Dineen, Eric Vang, Russel Haskin, Lisa A. M. Becker, Kazuaki Sekiguchi, Kisaburo Sakamoto, Carlos E. Ruiz on behalf of the Harmonization by Doing for Children Working Group

147. Cardiac Function by Magnetic Resonance Imaging in Coronary Artery Occlusions After Kawasaki Disease Hideyuki Nakaoka, Etsuko Tsuda, Yoshiaki Morita, Kenichi Kurosaki

148. Preoperative Threshold for Normalizing Right Ventricular Volume After Transcatheter Closure of Adult Atrial Septal Defect

Shintaro Umemoto, Ichiro Sakamoto, Kohtaro Abe, Ayako Ishikita, Yuzo Yamasaki, Ken-ichi Hiasa, Tomomi Ide, Hiroyuki Tsutsui

149. Pregnancy and Delivery in Patients With Repaired Congenital Heart Disease - A Retrospective Japanese Multicenter Study -

Shinichi Takatsuki, Yoshiyuki Furutani, Kei Inai, Tohru Kobayashi, Ryo Inuzuka, Tomomi Uyeda, Mitsuhiro Kamisago, Jun Muneuchi, Masahide Kaneko, Yasushi Misaki, Hiroshi Ono, Hitoshi Kato, Eriko Shimada, Tokuko Shinohara, Kenji Waki, Kenji Suda, Yasunobu Hayabuchi, Hirotaka Ohki, Reina Ishizaki, Jun Maeda, Hiroyuki Yamagishi

150. Discrepancy Between Pre- and Postnatal Diagnoses of Congenital Heart Disease and Impact on Neonatal Clinical Course - A Retrospective Study at a Japanese Tertiary Institution -

Kenichi Kurosaki, Masataka Kitano, Heima Sakaguchi, Isao Shiraishi, Naoko Iwanaga, Jun Yoshimatsu, Takaya Hoashi, Hajime Ichikawa, Satoshi Yasuda

Peripheral Vascular Disease (9)

151. Midterm Results of a Japanese Prospective Multicenter Registry of Heparin-Bonded Expanded Polytetrafluoroethylene Grafts for Above-the-Knee Femoropopliteal Bypass

Shintaro Shibutani, Hideaki Obara, Kentaro Matsubara, Naoki Toya, Naoko Isogai, Hidemitsu Ogino, Susumu Watada, Atsunori Asami, Toshifumi Kudo, Yuji Kanaoka, Naoki Fujimura, Hirohisa Harada, Hidetoshi Uchiyama, Yasunori Sato, Takao Ohki on behalf of the Japanese Bypass Registry Group, Tokyo, Japan

152. Diagnostic Performance of Dual-Layer Computed Tomography for Deep Vein Thrombosis in Indirect Computed Tomography Venography

Shota Tanoue, Takeshi Nakaura, Yuji Iyama, Ayumi Iyama, Yasunori Nagayama, Morikatsu Yoshida, Yasuyuki Yamashita

153. Comparative Analysis of the Paclitaxel-Eluting Peripheral Igaki-Tamai Stent and the Drug-Free Igaki-Tamai Stent Using Optical Coherence Tomography and Histological Analysis in a Porcine Iliac Artery Model

Kensuke Kuwabara, Kan Zen, Masaki Yashige, Nobuyasu Ito, Yoshito Kadoya, Noriyuki Wakana, Kenji Yanishi, Satoaki Matoba

154. Differences in Intravascular Ultrasound Measurement Values Between Treatment Modalities for Restenosis in Femoropopliteal Lesions Hideaki Aihara, Michiaki Higashitani, Hideyuki Takimura, Kazuki Tobita, Kentaro Jujo, Koji Hozawa, Tetsuo Yamaguchi, Yo Iwata, Hideo Tokuyama, Masayuki Sakurai, Naotaka Murata, Yo Fujimoto, Arifumi Kikuchi, Hiroshi Koganei, Akira Sato, Yuichi Noguchi, Masaki Ieda

155. Direct Oral Anticoagulant Therapy for Cancer-Associated Venous Thromboembolism in Routine Clinical Practice Yutaka Ogino, Tomoaki Ishigami, Yugo Minamimoto, Yuichiro Kimura, Eiichi Akiyama, Kozo Okada, Yasushi Matsuzawa, Nobuhiko Maejima, Noriaki Iwahashi, Kiyoshi Hibi, Masami Kosuge, Toshiaki Ebina, Toshiyuki Ishikawa, Kouichi Tamura, Kazuo Kimura

156. Influence of Self-Expanding Paclitaxel-Eluting Stent Sizing on Neointimal Hyperplasia in Superficial Femoral Artery Lesions

Kojiro Miki, Takamasa Tanaka, Koji Yanaka, Nagataka Yoshihara, Toshio Kimura, Takahiro Imanaka, Hirokuni Akahori, Masaharu Ishihara

157. Risk Factors for Progression of Distal Deep Vein Thrombosis

Shunichiro Fujioka, Hirotoki Ohkubo, Tadashi Kitamura, Toshiaki Mishima, Yoshihiko Onishi, Yuki Tadokoro, Haruna Araki, Takuya Matsushiro, Kazuki Yakuwa, Takashi Miyamoto, Shinzo Torii, Kagami Miyaji

158. Novel Evaluation Method for Lower Extremity Peripheral Artery Disease With Duplex Ultrasound - Usefulness of Acceleration Time Takeshi Yagyu, Sayaka Funabashi, Shuichi Yoneda, Teruo Noguchi, Satoshi Yasuda 
159. Angioscopic Assessments at 3 Months After Fluoropolymer-Based Paclitaxel-Eluting Stent Implantation for Femoropopliteal Endovascular Intervention

Takuya Tsujimura, Takayuki Ishihara, Osamu Iida, Yosuke Hata, Naoya Kurata, Mitsutoshi Asai, Masaharu Masuda, Shin Okamoto, Kiyonori Nanto, Takashi Kanda, Yasuhiro Matsuda, Toshiaki Mano

Preventive Medicine (3)

160. Fasting and Non-Fasting Triglycerides and Risk of Cardiovascular Events in Diabetic Patients Under Statin Therapy Hayato Tada, Akihiro Nomura, Kenichi Yoshimura, Hiroshi Itoh, Issei Komuro, Masakazu Yamagishi, Masayuki Takamura, Masa-aki Kawashiri

161. Effects of Bisoprolol Transdermal Patches for Prevention of Perioperative Myocardial Injury in High-Risk Patients Undergoing Non-Cardiac Surgery - Multicenter Randomized Controlled Study -

Hironobu Toda, Kazufumi Nakamura, Kazuyoshi Shimizu, Kentaro Ejiri, Takayuki Iwano, Toru Miyoshi, Koji Nakagawa, Masashi Yoshida, Atsuyuki Watanabe, Nobuhiro Nishii, Yukiko Hikasa, Masao Hayashi, Hiroshi Morita, Hiroshi Morimatsu, Hiroshi Ito on behalf of the MAMACARI Investigators

162. Circulating MicroRNA-423-3p Improves the Prediction of Coronary Artery Disease in a General Population - SixYear Follow-up Results From the China-Cardiovascular Disease Study -

Xin Wang, Ying Dong, Tian Fang, Xiaoxia Wang, Lu Chen, Congyi Zheng, Yuting Kang, Linlin Jiang, Xin You, Shujie Gai, Zengwu Wang, Huiqing Cao

Pulmonary Circulation (5)

163. Marked Reduction of Pulmonary Artery Pressure After Registration for Lung Transplantation Is Associated With Long-Term Survival in Patients With Pulmonary Arterial Hypertension - Cohort Study Satoshi Akagi, Hiromi Matsubara, Kazufumi Nakamura, Takahiro Oto, Kentaro Ejiri, Hiroshi Ito

164. Pristane/Hypoxia (PriHx) Mouse as a Novel Model of Pulmonary Hypertension Reflecting Inflammation and Fibrosis Hiroyoshi Mori, Tomohiko Ishibashi, Tadakatsu Inagaki, Makoto Okazawa, Takeshi Masaki, Ryotaro Asano, Yusuke Manabe, Keiko Ohta-Ogo, Masashi Narazaki, Hatsue Ishibashi-Ueda, Atsushi Kumanogoh, Yoshikazu Nakaoka

165. Effect of Oral Qing-Dai Medication on Pulmonary Arterial Pressure Levels in Patients With Ulcerative Colitis Yoshiyuki Orihara, Masanori Asakura, Nobuyuki Hida, Mikio Kawai, Toshiyuki Sato, Koichi Nishimura, Kumiko Masai, Yuki Matsumoto, Yoshitaka Okuhara, Akiko Goda, Tohru Masuyama, Shiro Nakamura, Masaharu Ishihara

166. Selexipag for Chronic Thromboembolic Pulmonary Hypertension in Japanese Patients - A Double-Blind, Randomized, Placebo-Controlled, Multicenter Phase II Study -

Nobuhiro Tanabe, Keiichi Fukuda, Hiromi Matsubara, Norifumi Nakanishi, Nobuhiro Tahara, Satoshi Ikeda, Takuya Kishi, Toru Satoh, Ken-ichi Hirata, Teruo Inoue, Hiroshi Kimura, Yoshiaki Okano, Osamu Okazaki, Masataka Sata, Ichizo Tsujino, Shuichi Ueno, Norikazu Yamada, Atsushi Yao, Takayuki Kuriyama

167. Risk Factors for Major Bleeding During Anticoagulation Therapy in Cancer-Associated Venous Thromboembolism - From the COMMAND VTE Registry -

Yuji Nishimoto, Yugo Yamashita, Kitae Kim, Takeshi Morimoto, Syunsuke Saga, Hidewo Amano, Toru Takase, Seiichi Hiramori, Maki Oi, Masaharu Akao, Yohei Kobayashi, Mamoru Toyofuku, Toshiaki Izumi, Tomohisa Tada, Po-Min Chen, Koichiro Murata, Yoshiaki Tsuyuki, Tomoki Sasa, Jiro Sakamoto, Minako Kinoshita, Kiyonori Togi, Hiroshi Mabuchi, Kensuke Takabayashi, Yusuke Yoshikawa, Hiroki Shiomi, Takao Kato, Takeru Makiyama, Koh Ono, Yukihito Sato, Takeshi Kimura on behalf of the COMMAND VTE Registry Investigators

Regenerative Medicine (1)

168. Long-Term Clinical Outcomes of Autologous Bone Marrow Mononuclear Cell Implantation in Patients With Severe Thromboangiitis Obliterans

Farina Mohamad Yusoff, Masato Kajikawa, Yuji Takaeko, Shinji Kishimoto, Haruki Hashimoto, Tatsuya Maruhashi, Yasuki Kihara, Ayumu Nakashima, Yukihito Higashi

Renal Disease (1)

169. Association of Dysfunction of Vascular Access for Hemodialysis With Major Adverse Cardiovascular Events - A Group-Based Trajectory Model Analysis -

Te-Hui Kuo, Chin-Li Lu, Ya-Hui Chang, Chung-Yi Li

Stroke (4)

170. Baseline Characteristics of Elderly Japanese Patients Aged $\geq 75$ Years With Non-Valvular Atrial Fibrillation and a History of Stroke - ANAFIE Registry Kodai Kanemaru, Takeshi Yoshimoto, Hiroshi Inoue, Takeshi Yamashita, Masaharu Akao, Hirotsugu Atarashi, Takanori Ikeda, Ken Okumura, Yukihiro Koretsune, Wataru Shimizu, Hiroyuki Tsutsui, Atsushi Hirayama, Masahiro Yasaka, Takenori Yamaguchi, Satoshi Teramukai, Tetsuya Kimura, Jumpei Kaburagi, Atsushi Takita, Kengo Kusano, Kazunori Toyoda for the All Nippon Atrial Fibrillation in the Elderly (ANAFIE) Group

171. Resting Heart Rate and In-Hospital Mortality in Acute Ischemic Stroke Patients With and Without Atrial Fibrillation Qiao Han, Chunyuan Zhang, Shoujiang You, Danni Zheng, Chongke Zhong, Hongli Dong, Xianhui Wang, Shaofang Pei, Yongjun Cao, Chun-Feng Liu

172. Age-Period-Cohort Analysis of Type-Specific Stroke Morbidity and Mortality in China Yuan Wang, Qin Peng, Jian Guo, Lihui Zhou, Wenli Lu 
173. Dabigatran vs. Aspirin for Secondary Prevention After Embolic Stroke of Undetermined Source - Japanese Subanalysis of the RE-SPECT ESUS Randomized Controlled Trial -

Kazunori Toyoda, Shinichiro Uchiyama, Yasushi Hagihara, Takahiro Kuwashiro, Takahisa Mori, Kenji Kamiyama, Yasuhisa Urano, Atsushi Taniguchi, Kenma Nozaki, Lisa Cronin, Claudia Grauer, Martina Brueckmann, Hans-Christoph Diener

Valvular Heart Disease (16)

174. Differences in Flow-Gradient Patterns Between Severe Bicuspid Aortic Stenosis and Severe Tricuspid Aortic Stenosis - Mechanistic Insight From Multimodal Imaging -

Darae Kim, Chi Young Shim, Young Jin Kim, Kyungsun Nam, Geu-Ru Hong, Seung Hyun Lee, Sak Lee, Jong-Won Ha

175. Age-Related Differences in the Effects of Initial Aortic Valve Replacement vs. Conservative Strategy on Long-Term Outcomes in Asymptomatic Patients With Severe Aortic Stenosis

Akihiro Kushiyama, Tomohiko Taniguchi,Takeshi Morimoto, Hiroki Shiomi, Kenji Ando, Norio Kanamori, Koichiro Murata, Takeshi Kitai, Yuichi Kawase, Chisato Izumi, Makoto Miyake, Hirokazu Mitsuoka, Masashi Kato, Yutaka Hirano, Shintaro Matsuda, Tsukasa Inada, Kazuya Nagao, Hiroshi Mabuchi, Yasuyo Takeuchi, Keiichiro Yamane, Mamoru Toyofuku, Mitsuru Ishii, Eri Minamino-Muta, Takao Kato, Moriaki Inoko, Tomoyuki Ikeda, Akihiro Komasa, Katsuhisa Ishii, Kozo Hotta, Nobuya Higashitani, Yoshihiro Kato, Yasutaka Inuzuka, Toshikazu Jinnai, Yuko Morikami, Naritatsu Saito, Kenji Minatoya, Takeshi Kimura on behalf of the CURRENT AS Registry Investigators

176. Incidence, Risk Factors and Subsequent Prognostic Impact of New-Onset Atrial Fibrillation in Infective Endocarditis Xue-biao Wei, Jie-leng Huang, Yuan-hui Liu, Chong-yang Duan, Ze-dazhong Su, Yu Wang, Dan-qing Yu, Ji-yan Chen

177. Importance of Preoperative Computed Tomography Assessment of the Membranous Septal Anatomy in Patients Undergoing Transcatheter Aortic Valve Replacement With a Balloon-Expandable Valve

Tomonori Miki, Keitaro Senoo, Takashi Ohkura, Yoshito Kadoya, Nobuyasu Ito, Kensuke Kuwabara, Naohiko Nakanishi, Kan Zen, Takeshi Nakamura, Tetsuhiro Yamano, Hirokazu Shiraishi, Takeshi Shirayama, Satoaki Matoba

178. Contemporary Outcomes of Surgical Aortic Valve Replacement in Japan

Yoshiyuki Tokuda, Hiroyuki Yamamoto, Hiroaki Miyata, Akihiko Usui, Noboru Motomura, The Japan Cardiovascular Surgery Database Organization

179. Comparison Between Healthcare-Associated and Community-Acquired Infective Endocarditis at Tertiary Care Hospitals in Japan

Hiroyuki Kiriyama, Masao Daimon, Koki Nakanishi, Hidehiro Kaneko, Tomoko Nakao, Ryoko Morimoto-Ichikawa, Sakiko Miyazaki, Hiroyuki Morita, Hiroyuki Daida, Issei Komuro

180. Transcatheter Aortic Valve Implantation vs. Surgical Aortic Valve Replacement for Severe Aortic Stenosis in RealWorld Clinical Practice

Yasuaki Takeji, Tomohiko Taniguchi, Takeshi Morimoto, Naritatsu Saito, Kenji Ando, Shinichi Shirai, Genichi Sakaguchi, Yoshio Arai, Yasushi Fuku, Yuichi Kawase, Tatsuhiko Komiya, Natsuhiko Ehara, Takeshi Kitai, Tadaaki Koyama, Shin Watanabe, Hirotoshi Watanabe, Hiroki Shiomi, Eri Minamino-Muta, Shintaro Matsuda, Hidenori Yaku, Yusuke Yoshikawa, Kazuhiro Yamazaki, Masahide Kawatou, Kazuhisa Sakamoto, Toshihiro Tamura, Makoto Miyake, Hisashi Sakaguchi, Koichiro Murata, Masanao Nakai, Norio Kanamori, Chisato Izumi, Hirokazu Mitsuoka, Masashi Kato, Yutaka Hirano, Tsukasa Inada, Kazuya Nagao, Hiroshi Mabuchi, Yasuyo Takeuchi, Keiichiro Yamane, Takashi Tamura, Mamoru Toyofuku, Mitsuru Ishii, Moriaki Inoko, Tomoyuki Ikeda, Katsuhisa Ishii, Kozo Hotta, Toshikazu Jinnai, Nobuya Higashitani, Yoshihiro Kato, Yasutaka Inuzuka, Yuko Morikami, Kenji Minatoya, Takeshi Kimura on behalf of the CURRENT AS Registry Investigators and K-TAVI Registry Investigators

181. Clinical Impact of New-Onset Left Bundle-Branch Block After Transcatheter Aortic Valve Implantation in the Japanese Population - A Single High-Volume Center Experience -

Kenichi Sasaki, Masaki Izumo, Shingo Kuwata, Yuki Ishibashi, Ryo Kamijima, Mika Watanabe, Toshiki Kaihara, Kazuaki Okuyama, Masashi Koga, Haruka Nishikawa, Yasuhiro Tanabe, Takumi Higuma, Yoshihiro J Akashi

182. Predictor and Mid-Term Outcome of Clinically Significant Thrombocytopenia After Transcatheter Aortic Valve Selection

Shinji Takahashi, Naoyuki Yokoyama, Yusuke Watanabe, Taiga Katayama, Hirofumi Hioki, Hirosada Yamamoto, Kazuo Kawasugi, Ken Kozuma

183. Elective Non-Cardiac Surgery in Patients With Severe Aortic Stenosis - Observations From the CURRENT AS Registry -

Tomohiko Taniguchi, Takeshi Morimoto, Hiroki Shiomi, Kenji Ando, Shinichi Shirai, Norio Kanamori, Koichiro Murata, Takeshi Kitai, Yuichi Kawase, Kazushige Kadota, Makoto Miyake, Chisato Izumi, Eri Minamino-Muta, Takao Kato, Katsuhisa Ishii, Kazuya Nagao, Naritatsu Saito, Kenji Minatoya, Takeshi Kimura on behalf of the CURRENT AS Registry Investigators

184. The Association of In-Hospital Transcatheter Aortic Valve Replacement Availability on Outcomes of Surgical Aortic Valve Replacement in Elderly Patients

Minoru Tabata, Hiraku Kumamaru, Aya Ono, Hiroaki Miyata, Yasunori Sato, Noboru Motomura 
185. Periprocedural Predictors of New-Onset Conduction Abnormalities After Transcatheter Aortic Valve Replacement Kensuke Matsushita, Mohamad Kanso, Mickael Ohana, Benjamin Marchandot, Marion Kibler, Joe Heger, Marilou Peillex, Antonin Trimaille, Sébastien Hess, Lelia Grunebaum, Antje Reydel, Fabien De Poli, Pierre Leddet, Jérôme Rischner, Philoktemon Plastaras, Laurence Jesel, Olivier Morel, Patrick Ohlmann

186. New-Generation Transcatheter Aortic Valves in Patients With Small Aortic Annuli - Comparison of Balloon- and Self-Expandable Valves in Asian Patients -

Kazuaki Okuyama, Masaki Izumo, Tomoki Ochiai, Shingo Kuwata, Toshiki Kaihara, Masashi Koga, Ryo Kamijima, Yuki Ishibashi, Yasuhiro Tanabe, Takumi Higuma, Raj Makkar, Takeshi Miyairi, Yoshihiro J Akashi

187. Effect of Immunosuppressive Therapy on Clinical Outcomes for Patients With Aortic Stenosis Following Transcatheter Aortic Valve Implantation

Toshiki Kaihara, Masaki Izumo, Haruka Kameshima, Yukio Sato, Shingo Kuwata, Masashi Koga, Mika Watanabe, Kazuaki Okuyama, Ryo Kamijima, Yuki Ishibashi, Yasuhiro Tanabe, Takumi Higuma, Tomoo Harada, Yoshihiro J. Akashi

188. Features of Lead-Induced Tricuspid Regurgitation in Patients With Heart Failure Events After Cardiac Implantation of Electronic Devices - A Three-Dimensional Echocardiographic Study -

Hideki Nakajima, Yoshihiro Seo, Tomoko Ishizu, Noriko Iida, Kimi Sato, Masayoshi Yamamoto, Tomoko Machino-Ohtsuka, Akihiko Nogami, Nobuyuki Ohte, Masaki Ieda

189. Long-Term Outcomes and Echocardiographic Data After Aortic Valve Replacement With a 17-mm Mechanical Valve

Homare Okamura, Daijiro Hori, Sho Kusadokoro, Makiko Mieno, Naoyuki Kimura, Koichi Yuri, Atsushi Yamaguchi

Vascular Biology and Vascular Medicine (1)

190. Changing Treatment Patterns in Patients With Venous Thromboembolism in Taiwan

Cheng-Han Lee, Ching-Chang Fang, Liang-Miin Tsai, Hui-Wen Lin, Po-Sheng Chen, Sheng-Hsiang Lin, Yi-Heng Li

Rapid Communications

1. Temporal Trends in Atherosclerotic Risk Factors in School Children - Findings From 20-Year Surveillance Daisuke Sueta, Eiichiro Yamamoto, Miyuki Sato, Takahiko Sato, Koichiro Fujisue, Yuichiro Arima, Seiji Takashio, Kenji Sakamoto, Hirofumi Soejima, Koichi Kaikita, Nobuyuki Shigaki, Yasuhiro Takasu, Kenichi Tsujita

2. Mechanical Circulatory Support Combined With Immunosuppression for the Treatment of Giant Cell Myocarditis - A Single-Center Experience in Japan -

Seiko Nakajima-Doi, Hiroki Mochizuki, Keiichiro Iwasaki, Kensuke Kuroda, Takuya Watanabe, Naoki Tadokoro, Atsushi Okada, Yasuhide Asaumi, Hideaki Kanzaki, Satsuki Fukushima, Osamu Seguchi, Masanobu Yanase, Chisato Izumi, Tomoyuki Fujita, Junjiro Kobayashi, Norihide Fukushima

3. Transesophageal Echocardiography During Cardiopulmonary Resuscitation (CPR-TEE) Kazumasa Orihashi

4. Angiotensin I Infusion Reveals Differential Effects of Angiotensin-Converting Enzyme in Aortic Resident Cells on Aneurysm Formation

Hisashi Sawada, Masayoshi Kukida, Xiaofeng Chen, Deborah A. Howatt, Jessica J. Moorleghen, Anju Balakrishnan, Congqing Wu, Alan Daugherty, Hong S. Lu

5. Roxadustat Markedly Reduces Myocardial Ischemia Reperfusion Injury in Mice Hiroko Deguchi, Masataka Ikeda, Tomomi Ide, Tomonori Tadokoro, Soichiro Ikeda, Kosuke Okabe, Akihito Ishikita, Keita Saku, Shouji Matsushima, Hiroyuki Tsutsui

6. Per-Vessel Level Analysis of Fractional Flow Reserve and Instantaneous Wave-Free Ratio Discordance - Insights From the AJIP Registry -

Takayuki Warisawa, Christopher M. Cook, Henry Seligman, James P. Howard, Yousif Ahmad, Christopher Rajkumar, Shunichi Doi, Masafumi Nakayama, Toru Tanigaki, Hiroyuki Omori, Akihiro Nakajima, Futoshi Yamanaka, Sonoka Goto, Yohei Yakuta, Kenichi Karube, Teruyoshi Uetani, Yuetsu Kikuta, Yasutsugu Shiono, Yoshiaki Kawase, Hidetaka Nishina, Sunao Nakamura, Javier Escaned, Yoshihiro J. Akashi, Hitoshi Matsuo, Justin E. Davies

7. Coronavirus Disease 2019 (COVID-19) Information for Cardiologists - Systematic Literature Review and Additional Analysis -

Tadafumi Sugimoto, Atsushi Mizuno, Takuya Kishi, Naoya Ito, Chisa Matsumoto, Memori Fukuda, Nobuyuki Kagiyama, Tatsuhiro Shibata, Takashi Ohmori, Shogo Oishi, Jun Fuse, Keisuke Kida, Fujimi Kawai, Mari Ishida, Shoji Sanada, Issei Komuro, Koichi Node

8. NT5E Genetic Mutation Is a Rare But Important Cause of Intermittent Claudication and Chronic Limb-Threatening Ischemia

Nobuyoshi Azuma, Tetsuro Uchida, Shinsuke Kikuchi, Mitsuaki Sadahiro, Tsunehiro Shintani, Kumiko Yanagi, Ryuji Higashita, Atsushi Yamashita, Yoshio Makita, Tadashi Kaname 
9. Safety and Efficacy of Human Muse Cell-Based Product for Acute Myocardial Infarction in a First-in-Human Trial Toshiyuki Noda, Kazuhiko Nishigaki, Shinya Minatoguchi

10. Recommendations for Maintaining the Cardiovascular Care System Under the Conditions of the COVID-19 Pandemic - 1st Edition, April 2020 -

Takuya Kishi, Atsushi Mizuno, Mari Ishida, Chisa Matsumoto, Memori Fukuda, Shoji Sanada, Naoya Itoh, Hideaki Oka, Koichi Node, Issei Komuro on behalf of the Collaborators and Advisors of the COVID-19 Task Force Mission Team of the Japanese Circulation Society, and Directors of the Japanese Circulation Societies

11. Human-Induced Pluripotent Stem Cell-Derived Cardiomyocytes Platform to Study SARS-CoV-2 Related Myocardial Injury

Chun-Ka Wong, Hayes Kam-Hei Luk, Wing-Hon Lai, Yee-Man Lau, Ricky Ruiqi Zhang, Antonio Cheuk-Pui Wong, George Chi-Shing Lo, Kwok-Hung Chan, Ivan Fan-Ngai Hung, Hung-Fat Tse, Patrick Chiu-Yat Woo, Susanna Kar-Pui Lau, Chung-Wah Siu

Images in Cardiovascular Medicine

1. Angioscopy of In-Stent Restenosis After Second-Generation Drug-Eluting Stent Implantation - Three HighResolution Coronary Angioscopy Observations -

Yuhei Nojima, Hidenori Adachi, Madoka Ihara, Tetsuya Kurimoto, Shinsuke Nanto

2. Coronary Artery Features of Immunoglobulin-G4-Related Coronary Periarteritis With Multi-Modality Visualization Mitsumasa Sudo, Daisuke Fukamachi, Shunichi Yoda, Yasuo Okumura

3. Pathology of Coronary Artery Embolism Derived From Mural Thrombus of Left Ventricle

Toru Miyoshi, Hiroyuki Hao, Akira Oshita, Hideo Kawakami, Toshiharu Maeda, Hiroshi Matsuoka
4. MitraClip Therapy for Healed Infective Endocarditis - How Long Should We Wait After Active Infection? Yuta Kato, Makoto Amaki, Hideaki Kanzaki, Yu Kataoka, Atsushi Okada, Koji Miyamoto, Yasuhiro Hamatani, Masashi Amano, Hiroyuki Takahama, Takuya Hasegawa, Yorihiko Matsumoto, Tomoyuki Fujita, Junjiro Kobayashi, Hatsue Ishibashi-Ueda, Satoshi Yasuda, Chisato Izumi

(1)

5. Successful Disruption of Massive Calcified Nodules Using Novel Shockwave Intravascular Lithotripsy Takayuki Warisawa, Carlos H. Salazar, Nieves Gonzalo, Yoshihiro J. Akashi, Javier Escaned

6. Hitting Two Birds With One Stone - Pre-Existing Paravalvular Leak Closed by Valve-in-Valve Implantation Abdisamad A. Osoble, Arun Kumar, Thomas Bartel

7. Cardiac Rupture Due to Side Branch Occlusion After Stent Implantation - The Crime of Jailed Stent Kumiko Asakura, Taku Homma, Naotaka Akutsu, Daisuke Fukamachi, Shunsuke Ozaki, Hiroshi Ohta, Yasuo Okumura, Hiroyuki Hao

8. Keloid as an Underrecognized Potential Risk Factor for Post-Procedural Pulmonary Vein Stenosis Hiro Yamasaki, Tomoya Hoshi, Kazutaka Aonuma, Akihiko Nogami, Masaki Ieda

9. Acute Popliteal Artery Thrombosis After Arthroscopic Anterior Cruciate Ligament Reconstruction Kanichi Otowa, Yoshinobu Maruhashi, Akio Chikata, Michiro Maruyama, Kazuo Usuda

10. Extremely Rare Case of Submitral Aneurysm With Left Atrium Communication in a Japanese Patient Yutaka Matsuhiro, Ryu Shutta, Kyosuke Yanagawa, Hitoshi Nakamura, Koji Yasumoto, Masaki Tsuda, Akihiro Tanaka, Naotaka Okamoto, Yasuharu Matsunaga-Lee, Masamichi Yano, Masaki Yamato, Yasuyuki Egami, Masami Nishino, Jun Tanouchi

11. Importance of Terminating Pulmonary Vein Fibrillation for Complete Pulmonary Vein Isolation Songwen Chen, Juan Xu, Xiaoyu Wu, Shaowen Liu

12. Coronary Artery Injury Caused by Leadless Pacemaker Implantation Hidehira Fukaya, Jun Kishihara, Naruya Ishizue, Ryo Kameda, Takao Shimohama, Yuki Arakawa, Ryo Nishinarita, Ai Horiguchi, Jun Oikawa, Shinichi Niwano, Junya Ako

13. Role of Invasive Hemodynamic Assessment During Trans-Catheter Paravalvular Leak Intervention Ashish H. Shah, Nasir Shaikh, Amir Ravandi, Malek Kass

14. Subclinical Calcified Nodules in Saphenous Vein Graft on High-Resolution Coronary Angioscopy Yohei Sumino, Tomoyo Sugiyama, Masahiro Hoshino, Yoshihisa Kanaji, Taishi Yonetsu, Tsunekazu Kakuta Effect of Deep Breathing in a Patient With Single Coronary Artery Compressed by Aorta and Pulmonary Artery - Intravascular Ultrasound and Fractional Flow Reserve Assessment Yusuke Nakano, Katsuhisa Waseda, Atomu Tajima, Hirohiko Ando, Hiroaki Takashima, Tetsuya Amano

16. Detection of Spontaneous Subintimal Hemorrhage on Angioscopy 1 Year Before Acute Aortic Dissection Satoru Takahashi, Sei Komatsu, Chikao Yutani, Mitsuhiko Takewa, Tomoki Ohara, Kazuhisa Kodama So Ikebe, Seiji Takashio, Masato Nishi, Mami Morioka, Kenichi Tsujita

18. Endoscopic Findings of an Excessively Compressed Interwoven Nitinol Supera Stent Makoto Sugihara, Shin-ichiro Miura

\section{Transthyretin Amyloid Cardiomyopathy Diagnosed on Incidental Myocardial Uptake During Bone Scintigraphy} Optical Coherence Tomography-Guided Stent Implantation for Woven Coronary Artery Anomaly Yusuke Uemura, Akihito Tanaka, Kenji Takemoto, Hideki Ishii, Toyoaki Murohara, Masato Watarai 
20. An Autopsy Case of Arrhythmogenic Right Ventricular Cardiomyopathy - Radiological/Pathological Comparison Kenta Uto, Michinobu Nagao, Noriko Kikuchi, Hiromi Onizuka, Saeko Yoshizawa, Nobuhisa Hagiwara, Hideaki Oda

21. Pace-and-Ablate Technique for Atrial Tachycardia Originating From the Left Atrial Appendage Taiki Sato, Koji Miyamoto, Tatsuya Nishii, Kengo Kusano

Thological Features of Lamin Cardiomyopathy

Hiroaki Kawano, Takashi Ishimatsu, Koichi Kawamura, Mitsuaki Ishijima, Tomayoshi Hayashi, Taisuke Ishikawa, Naomasa Makita, Koji Maemura

23. Myocarditis in a Patient With Clinically Amyopathic Dermatomyositis

Hiroaki Kawano, Naoe Kinoshita, Seiya Izumida, Toshimasa Shimizu, Masataka Umeda, Koji Maemura

24. Clinical Implications of Cardiac Magnetic Resonance Imaging for Identifying Culprit Lesions in Patients With Suspected Multivessel Acute Myocardial Infarction

Riku Arai, Akimasa Yamada, Saki Mizobuchi, Naotaka Akutsu, Toshihiko Nishida, Daisuke Fukamachi, Yasuo Okumura

25. Long-Term Follow-up of a Coronary Artery Aneurysm After Biodegradable-Polymer Sirolimus-Eluting Stent Implantation

Toru Yoshizaki, Aki Kobayashi, Takamitsu Nakamura, Jun-ei Obata, Kiyotaka Kugiyama

26. Atrial Septal Hematoma After Transcatheter Aortic Valve Implantation Subaru Tanabe, Yusuke Nakano, Hirohiko Ando, Hirofumi Ohashi, Kentaro Mukai, Tetsuya Amano

27. Slowly Developed Coronary Artery Aneurysms in a Patient With Probable Kawasaki Disease Yukiko Mizutani, Tetsuya Ishikawa, Shingo Watanabe, Sayuki Kobayashi, Isao Taguchi

28. Overhanging Bioresorbable Vascular Scaffold Observed on Angioscopy 1 Year After Implantation Hiroyuki Inoue, Atsunori Okamura, Mutsumi Iwamoto, Akinori Sumiyoshi, Kota Tanaka, Kohtaro Takayasu, Katsutoshi Kawamura, Yasushi Koyama, Koichi Inoue, Katsuomi Iwakura, Kenshi Fujii, Yasushi Sakata, Hiroyuki Nagai

29. Detailed Characterization of Vulnerable Plaque Morphology by High-Resolution Intravascular Ultrasound Hirohiko Ando, Hirofumi Ohashi, Yusuke Nakano, Hiroaki Takashima, Tetsuya Amano

30. Pulsatile Strangulation of the Aorta - A Rare Presentation and Etiology of Infective Endocarditis Wei-Ting Chang, Zhih-Cherng Chen

31. Vasculopathy After Transradial Catheterization in a Scleroderma Patient Takenobu Shimada, Seiji Habara, Hiroyuki Tanaka, Kazushige Kadota

2. Missing P Wave on Electrocardiogram in Hypermagnesemia Hiroaki Nishioka

33. de Winter Electrocardiogram Pattern Due to Vasospastic Angina Hirohiko Ando, Masahiro Shimoda, Hirofumi Ohashi, Yusuke Nakano, Hiroaki Takashima, Tetsuya Amano

34. High-Resolution Angioscopic Observation of Deep Vein Thrombosis After Catheter-Directed Venous Thrombolysis Atsutoshi Hatada, Yusaku Takagaki, Shuji Yamamoto, Yoshitaka Okamura, Yoshiharu Nishimura

35. ${ }^{123}$ I- $\beta$-Methyl Iodophenyl Pentadecanoic Acid and ${ }^{201}$ Thallium Dual Myocardial Single-Photon Emission Computed Tomography (BMIPP/Tl SPECT) Detection of Myocardial Damage of Systemic Sclerosis Hiroaki Kawano, Takashi Kudo, Koji Maemura

36. Glucocorticoid-Induced Bacterial Endocarditis in COVID-19 Pneumonia - Something to Be Concerned About? Valentina Regazzoni, Marco Loffi, Alberto Garini, Gian Battista Danzi

37. Diagnosis of Spontaneous Reperfusion Case in Acute Myocardial Infarction by Fusion of Technetium-99 m Pyrophosphate/Thallium-201 Dual-Isotope Single Photon Emission CT Imaging and Contrast-Enhancement Coronary CT

Kansuke Ito, Ken Kato, Kenji Fukushima, Hiroyuki Tanaka

38. Fractured Sternal Wire With Distal Migration and Penetration of the Right Ventricle Ichitaro Abe, Miho Miyoshi, Tomoko Fukuda, Taisuke Harada, Keisuke Yonezu, Hidefumi Akioka, Kunio Yufu, Naohiko Takahashi

39. Transapical Off-Pump Mitral Valve Repair With NeoChord Implantation - First 2 Cases in Japan Daisuke Yoshioka, Kazuo Shimamura, Kaoruko Sengoku, Koichi Toda, Takaaki Samura, Shigeru Miyagawa, Yasushi Sakata, Yoshiki Sawa

40. Exertional Angina in a Child With Single Right Coronary Artery and Septal Course of the Left Anterior Descending Artery - A Rare Cause of Angina -

Yoshihiko Kodama, Hiroki Ezaki, Yuichi Ishikawa, Koichi Sagawa

41. High-Resolution Mapping to Unmask and Localize Presystolic Purkinje Potentials During Idiopathic VerapamilSensitive Left Anterior Papillary Muscle Fascicular-Purkinje Ventricular Tachycardia Itsuro Morishima, Akihiko Nogami, Yasunori Kanzaki

42. Mechanism of Early-Diastolic Mitral Regurgitation Michito Murayama, Hiroyuki Iwano, Miwa Sarashina, Toshihisa Anzai

43. Angioscopic Findings of Deep Vein Thrombosis Treated by Balloon Angioplasty With Catheter-Directed Thrombolysis Naoki Kubota, Kazuyuki Ozaki, Makoto Hoyano, Tohru Minamino 
44. Avulsion of Previously Implanted Stent by Directional Coronary Atherectomy - Intravascular Ultrasound and Angioscopic Evaluation -

Yasuyuki Egami, Ryu Shutta, Kohei Ukita, Akito Kawamura, Hitoshi Nakamura, Yutaka Matsuhiro, Koji Yasumoto, Masaki Tsuda, Naotaka Okamoto, Akihiro Tanaka, Yasuharu Matsunaga-Lee, Masamichi Yano, Masami Nishino, Jun Tanouchi

45. Efficacy of Excimer Laser Coronary Angioplasty for Repeated In-Stent Restenosis With Neointimal Hyperplasia and Underexpanded Stent

Daigo Hiraya, Akira Sato, Taikan Terauchi, Hiroaki Watabe, Tomoya Hoshi, Masaki Ieda

46. Delayed Cardiac Tamponade Due to Possible Left Atrial Injury Following Self-Expandable Transcatheter Aortic Valve Implantation

Masaki Tsuda, Ryu Shutta, Akito Kawamura, Kohei Ukita, Hitoshi Nakamura, Yutaka Matsuhiro, Koji Yasumoto, Naotaka Okamoto, Akihiro Tanaka, Yasuharu Matsunaga-Lee, Masamichi Yano, Yasuyuki Egami, Masami Nishino, Jun Tanouchi

47. Symptomatic Right Ventricular Outflow Tract Obstruction Due to Sinus of Valsalva Aneurysm Akihito Saito, Katsura Soma, Akihiro Masuzawa, Satoshi Hatsuse, Yukiteru Nakayama, Eisuke Amiya, Atsushi Yao, Yasutaka Hirata, Issei Komuro

\section{JCS Guidelines}

1. JCS 2017 Guideline on Management of Vasculitis Syndrome - Digest Version -

Mitsuaki Isobe, Koichi Amano, Yoshihiro Arimura, Akihiro Ishizu, Shuichi Ito, Shinya Kaname, Shigeto Kobayashi, Yoshinori Komagata, Issei Komuro, Kimihiro Komori, Kei Takahashi, Kazuo Tanemoto, Hitoshi Hasegawa, Masayoshi Harigai, Shouichi Fujimoto, Tatsuhiko Miyazaki, Tetsuro Miyata, Hidehiro Yamada, Akitoshi Yoshida, Takashi Wada, Yoshinori Inoue, Haruhito A. Uchida, Hideki Ota, Takahiro Okazaki, Mitsuho Onimaru, Tamihiro Kawakami, Reiko Kinouchi, Atsushi Kurata, Hisanori Kosuge, Ken-ei Sada, Kunihiro Shigematsu, Eiichi Suematsu, Eijun Sueyoshi, Takahiko Sugihara, Hitoshi Sugiyama, Mitsuhiro Takeno, Naoto Tamura, Michi Tsutsumino, Hiroaki Dobashi, Yoshikazu Nakaoka, Kenji Nagasaka, Yasuhiro Maejima, Hajime Yoshifuji, Yoshiko Watanabe, Shoichi Ozaki, Takeshi Kimura, Hiroshi Shigematsu, Keiko Yamauchi-Takihara, Toyoaki Murohara, Shin-ichi Momomuraon behalf of the JCS Joint Working Group

2. JCS 2020 Guideline Focused Update on Antithrombotic Therapy in Patients With Coronary Artery Disease Masato Nakamura, Kazuo Kimura, Takeshi Kimura, Masaharu Ishihara, Fumiyuki Otsuka, Ken Kozuma, Masami Kosuge, Toshiro Shinke, Yoshihisa Nakagawa, Masahiro Natsuaki, Satoshi Yasuda, Takashi Akasaka, Shun Kohsaka, Kazuo Haze, Atsushi Hirayama

3. JCS/JSCS 2020 Guideline on Diagnosis and Management of Cardiovascular Sequelae in Kawasaki Disease Ryuji Fukazawa, Junjiro Kobayashi, Mamoru Ayusawa, Hiromichi Hamada, Masaru Miura, Yoshihide Mitani, Etsuko Tsuda, Hiroyuki Nakajima, Hiroyuki Matsuura, Kazuyuki Ikeda, Kazuhiko Nishigaki, Hiroyuki Suzuki, Kei Takahashi, Kenji Suda, Hiroshi Kamiyama, Yoshihiro Onouchi, Tohru Kobayashi, Hiroyoshi Yokoi, Kisaburo Sakamoto, Masami Ochi, Soichiro Kitamura, Kenji Hamaoka, Hideaki Senzaki, Takeshi Kimura on behalf of the Japanese Circulation Society Joint Working Group

4. JCS 2020 Guideline on Diagnosis and Treatment of Cardiac Amyloidosis

Hiroaki Kitaoka, Chisato Izumi, Yasuhiro Izumiya, Takayuki Inomata, Mitsuharu Ueda, Toru Kubo, Jun Koyama, Motoaki Sano, Yoshiki Sekijima, Nobuhiro Tahara, Nobuhiro Tsukada, Kenichi Tsujita, Hiroyuki Tsutsui, Takeshi Tomita, Masashi Amano, Jin Endo, Atsushi Okada, Seitaro Oda, Seiji Takashio, Yuichi Baba, Yohei Misumi, Masahide Yazaki, Toshihisa Anzai, Yukio Ando, Mitsuaki Isobe, Takeshi Kimura, Keiichi Fukuda on behalf of the Japanese Circulation Society Joint Working Group

5. JCS/JSCS/JATS/JSVS 2020 Guidelines on the Management of Valvular Heart Disease

Chisato Izumi, Kiyoyuki Eishi, Kyomi Ashihara, Takeshi Arita, Yutaka Otsuji, Takashi Kunihara, Tatsuhiko Komiya, Toshihiko Shibata, Yoshihiro Seo, Masao Daimon, Shuichiro Takanashi, Hiroyuki Tanaka, Satoshi Nakatani, Hiroshi Ninami, Hiroyuki Nishi, Kentaro Hayashida, Hitoshi Yaku, Junichi Yamaguchi, Kazuhiro Yamamoto, Hiroyuki Watanabe, Yukio Abe, Makoto Amaki, Masashi Amano, Kikuko Obase, Minoru Tabata, Takashi Miura, Makoto Miyake, Mitsushige Murata, Nozomi Watanabe, Takashi Akasaka, Yutaka Okita, Takeshi Kimura, Yoshiki Sawa, Kiyoshi Yoshidaon behalf of the Japanese Circulation Society Joint Working Group

\section{Letters to the Editor}

1. Utility of Kumamoto Criteria in Diagnosing Transthyretin Cardiac Amyloidosis in Real-World Practice (Letter) Toru Kubota, Genya Sunagawa, Daisuke Nagatomo, Yoshitomo Tsutsui, Hiroyuki Tsutsui (Reply) Seiji Takashio, Kyohei Marume, Masato Nishi, Koichi Kaikita, Kenichi Tsujita

2. Earlier Diagnosis of Peripartum Cardiomyopathy (PPCM) (Letter) James D. Fett

3. Is High Heart Rate Always Harmful to Heart Failure Patients? (Letter) Sho Okada, Togo Iwahana, Yoshio Kobayashi (Reply) Hiroyuki Tsutsui, Tomomi Ide, Yasushi Kawasaki 
4. Cell Therapy Strategies With No Safety Concerns and Demonstrated Benefits Warrant Study (Letter) Carl J. Pepine, Amish N. Raval

(Reply) Kenta Nakamura, Charles E. Murry

….... 2120

….... 2122

\section{Retraction}

1. (Retraction Request) Electrophysiological Characteristics and Radiofrequency Catheter Ablation Treatment of Idiopathic Ventricular Arrhythmias Successfully Ablated From the Ostium of the Coronary Sinus Yoshiaki Yui, Yukio Sekiguchi, Akihiko Nogami, Hiro Yamasaki, Takeshi Machino, Kenji Kuroki, Miyako Igarashi, Kazutaka Aonuma

2. (Retraction Request) Efficacy of Intensive Radiofrequency Energy Delivery to the Localized Dense Scar Area in Post-Infarction Ventricular Tachycardia Ablation - A Comparative Study With Standard Strategy Targeting the Infarcted Border Zone Kenji Kuroki

\section{Corrigendum}

1. Baseline Demographics and Clinical Characteristics in the All Nippon AF in the Elderly (ANAFIE) Registry Yukihiro Koretsune, Takeshi Yamashita, Masaharu Akao, Hirotsugu Atarashi, Takanori Ikeda, Ken Okumura, Wataru Shimizu, Hiroyuki Tsutsui, Kazunori Toyoda, Atsushi Hirayama, Masahiro Yasaka, Takenori Yamaguchi, Satoshi Teramukai, Tetsuya Kimura, Jumpei Kaburagi, Atsushi Takita, Hiroshi Inoue

2. Influence of Preadmission Frailty on Short- and Mid-Term Prognoses in Octogenarians With ST-Elevation Myocardial Infarction

Naoki Yoshioka, Kensuke Takagi, Itsuro Morishima, Yasuhiro Morita, Yusuke Uemura, Yosuke Inoue, Norio Umemoto, Naoki Shibata, Yosuke Negishi, Ruka Yoshida, Akihito Tanaka, Hideki Ishii, Toyoaki Murohara on behalf of the N-Registry Investigators

3. Acute Coronary Syndrome With Unprotected Left Main Coronary Artery Culprit - An Observation From the AOILMCA Registry -

Hirooki Higami, Mamoru Toyofuku, Takeshi Morimoto, Masanobu Ohya, Yasushi Fuku, Kyohei Yamaji, Hiromi Muranishi, Yuhei Yamaji, Koji Nishida, Daisuke Furukawa, Tomohisa Tada, Euihong Ko, Kenji Ando, Hiroki Sakamoto, Takashi Tamura, Kazuya Kawai, Kazushige Kadota, Takeshi Kimura on behalf of the AOI-LMCA Stenting Registry Investigators

4. Association of Albuminuria With White Matter Hyperintensities Volume on Brain Magnetic Resonance Imaging in Elderly Japanese - The Hisayama Study -

Keisuke Yamasaki, Jun Hata, Yoshihiko Furuta, Naoki Hirabayashi, Tomoyuki Ohara, Daigo Yoshida, Yoichiro Hirakawa, Toshiaki Nakano, Takanari Kitazono, Toshiharu Ninomiya

5. Age-Dependent Clinical and Genetic Characteristics in Japanese Patients with Arrhythmogenic Right Ventricular Cardiomyopathy/Dysplasia

Seiko Ohno, Iori Nagaoka, Megumi Fukuyama, Hiromi Kimura, Hideki Itoh, Takeru Makiyama, Akihiko Shimizu, Minoru Horie

6. Genetic Background of Catecholaminergic Polymorphic Ventricular Tachycardia in Japan

Mihoko Kawamura, Seiko Ohno, Nobu Naiki, Iori Nagaoka, Kenichi Dochi, Qi Wang, Kanae Hasegawa, Hiromi Kimura, Akashi Miyamoto, Yuka Mizusawa, Hideki Itoh, Takeru Makiyama, Naokata Sumitomo, Hiroya Ushinohama, Kotaro Oyama, Nobuyuki Murakoshi, Kazutaka Aonuma, Hitoshi Horigome, Takafumi Honda, Masao Yoshinaga, Makoto Ito, Minoru Horie

7. Impact of Chronic Kidney Disease Classification on New-Onset Atrial Fibrillation in the General Population - The TAMA MED Project-AF and CKD -

Eitaro Kodani, Tomohiro Kaneko, Hitomi Fujii, Hiroyuki Nakamura, Hajime Sasabe, Yutaka Tamura, Wataru Shimizu 